\title{
An Interactive Design Space for Wearable Displays
}

\author{
Florian Heller \\ Hasselt University - tUL - Flanders \\ Make \\ Diepenbeek, Belgium \\ florian.heller@gmail.com
}

\author{
Kashyap Todi \\ Aalto University \\ Helsinki, Finland \\ kashyap.todi@gmail.com
}

\author{
Kris Luyten \\ Hasselt University - tUL - Flanders \\ Make \\ Diepenbeek, Belgium \\ kris.luyten@uhasselt.be
}

\begin{abstract}
The promise of on-body interactions has led to widespread development of wearable displays. They manifest themselves in highly variable shapes and form, and are realized using technologies with fundamentally different properties. Through an extensive survey of the field of wearable displays, we characterize existing systems based on key qualities of displays and wearables, such as location on the body, intended viewers or audience, and the information density of rendered content. We present the results of this analysis in an open, web-based interactive design space that supports exploration and refinement along various parameters. The design space, which currently encapsulates 129 cases of wearable displays, aims to inform researchers and practitioners on existing solutions and designs, and enable the identification of gaps and opportunities for novel research and applications. Further, it seeks to provide them with a thinking tool to deliberate on how the displayed content should be adapted based on key design parameters. Through this work, we aim to facilitate progress in wearable displays, informed by existing solutions, by providing researchers with an interactive platform for discovery and reflection.
\end{abstract}

\section{CCS CONCEPTS}

- Human-centered computing $\rightarrow$ Displays and imagers; Ubiquitous and mobile computing.

\section{KEYWORDS}

Wearable Computing, Displays, Survey, Design Space

\section{ACM Reference Format:}

Florian Heller, Kashyap Todi, and Kris Luyten. 2021. An Interactive Design Space for Wearable Displays. In 23rd International Conference on Mobile Human-Computer Interaction (MobileHCI '21), September 27-October 1, 2021, Toulouse \& Virtual, France. ACM, New York, NY, USA, 14 pages. https: //doi.org/10.1145/3447526.3472034

\section{INTRODUCTION}

On-body interactions have been of great interest to the research community and industry for a long time, resulting in sophisticated solutions that either take advantage of the body as an interactive surface $[58,155]$, make clothing interactive $[78,119]$, or integrate

Permission to make digital or hard copies of all or part of this work for personal or classroom use is granted without fee provided that copies are not made or distributed for profit or commercial advantage and that copies bear this notice and the full citation on the first page. Copyrights for components of this work owned by others than the author(s) must be honored. Abstracting with credit is permitted. To copy otherwise, or republish, to post on servers or to redistribute to lists, requires prior specific permission and/or a fee. Request permissions from permissions@acm.org.

MobileHCI '21, September 27-October 1, 2021, Toulouse \& Virtual, France

(c) 2021 Copyright held by the owner/author(s). Publication rights licensed to ACM.

ACM ISBN 978-1-4503-8328-8/21/09..\$15.00

https://doi.org/10.1145/3447526.3472034

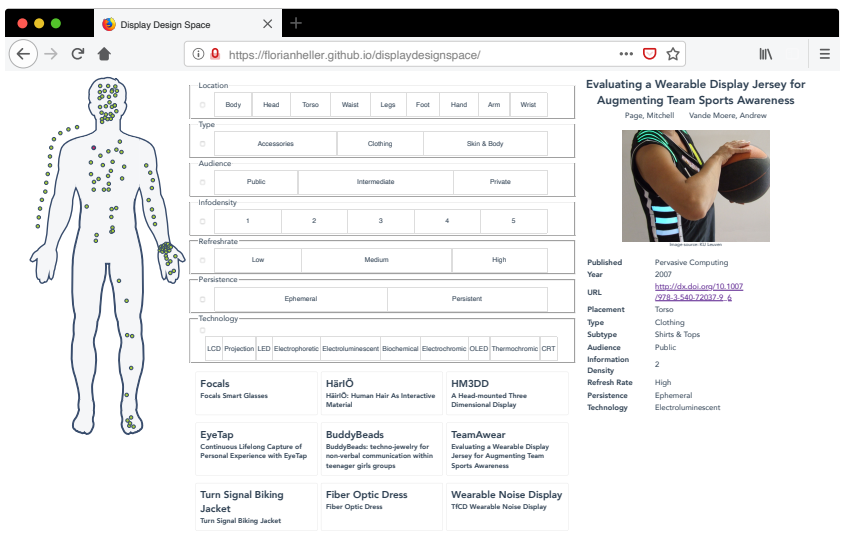

Figure 1: This paper presents a taxonomy for wearable displays, and an interactive design space. The web-based tool supports exploration through an interactive visualization and categorical filters.

computational power within wearable accessories. In many cases, input technologies and interactions have been direct adaptations of concepts from the desktop and mobile computers to the textile and wearable context. The output direction, i.e., incorporating lightemitting displays into the wearable ecosystem is more challenging. The resolution of LED-based displays is limited, especially when integrated in textile, because of manufacturing characteristics such as the minimal distance between two conductive threads. There have been attempts to integrate flexible displays into wearables, such as a complete dress capable of altering its visual appearance [36], but the garment is still far from normal clothing in terms of flexibility, breathability, fashionabilty, or washability [46, 89]. Turning fabric into a display itself is the ultimate goal, and while functional prototypes exist, their manufacturing is still a complex process [152]. Nevertheless, a multitude of wearable display prototypes and technologies have emerged over the last decades, ranging from simple LEDs to high-resolution output. Work on wearable displays crosses the boundaries between makers, researchers, and chemical and electronics industry, which makes it difficult to quickly get an overview of the field.

Design spaces, surveys, and taxonomies in HCI provide a valuable method for synthesising knowledge, summarising key findings, highlighting challenges, and identifying opportunities. They have been used previously to, for example, systematically analyse input devices [18], study specific interaction modalities [13], and to study the application of specific methods [116]. In this paper, we survey various sources for wearable and textile displays and place them into a design space based on dimensions 
of technical integration, wearability, placement on body, and target audience. We scope our survey to two-dimensional pixel-based displays, and refer the readers to previous literature for a survey of shape-changing displays [126]. We discuss different categories of information or data typically displayed on wearable displays, and provide illustrative examples. The result of this classification is a web-based interactive design space, freely accessible at: https://florianheller.github.io/displaydesignspace . One of the key objectives of our design space is to provide researchers and practitioners with an overarching view of existing projects. To this end, our web platform positions the surveyed works onto appropriate locations on an interactive body silhouette, and lists them in entirety in a table. Further, we aim to facilitate fine-grained and refined exploration through filters for all the dimensions of the classification. The second objective of our work is to provide researchers and designers with a tool for generating ideas and new solutions. As such, our design space can enable discovery by highlighting blind spots (currently unexplored areas), and by pointing out possibilities for transfer of knowledge between dimensions. Finally, we believe that the design space can be used as a problem-solving tool while designing or adapting content for wearable displays. It can provide researchers and practitioners with hints on how to adapt their content for different categories, or it could be applied towards developing automated approaches for cross-display applications, to adapt content based on context of use.

\section{DISPLAY TECHNOLOGIES: AN OVERVIEW}

To create wearable visual output, a multitude of technologies are available, ranging from off-the-shelf components to high-end materials only available in chemistry labs. Here, we provide a brief overview of commonly-used technologies, and discuss their use in the wearable domain.

Display technologies can be broken down into two categories: 1) ones that emit light on their own, e.g., LEDs, Electroluminescent dyes, OLED; and 2) those that alter how light is reflected or let through, e.g., thermochromic and electrochromic inks, electrophoretic (E Ink) displays.

Light-emitting diodes (LEDs) are easily sourceable components and, therefore, a popular form to create wearable displays. Toolkits like Adafruit Flora [1] or Lillypad provide easy to connect LED modules in various colors. However, with increasing number, addressing and connecting individual LEDs becomes problematic in a textile context, e.g., because the minimal distance between two lines of conductive yarn is substantially larger than on normal, rigid or flexible circuit boards. To increase the density of LED arrays, these need to be placed on fixed or flexible PCB substrates, which reduces their wearability. Instead of using LEDs as point light sources, their output can be redirected using optical fibers [9] and diffusors. Placing LEDs on a garment also often happens in a more organic fashion than in electronics design, which raises the issue of calibration in order to control what is shown on the display [24].

Liquid crystal displays (LCDs) are most common in non-wearable systems, ranging from single distinct elements that can be enabled or disables, up to high-resolution computer screens. With the exception of OLED LCDs, they do not emit any light, but instead, need a backlight to be readable under low environmental light conditions.
Electroluminscent (EL) coatings can be applied directly onto textile materials [30, 169], reducing the impact on wearability [22]. By applying an alternating electric field, this coating lights up in a specific color, determined by the chemical composition of the dye. EL light sources have a low power consumption and fast reaction times, but can only emit a single color. Furthermore, because of the AC fields, wiring is more complex than with LEDs which can share a common ground wire. Nevertheless, EL displays can be created with simple techniques like screen printing [31].

The remaining technologies all do not emit light on their own, but either selectively cover a background light source (transmissive), or change how incoming light is reflected (reflective).

Electrochromic (EC) inks, e.g., the commercially available ynvisible ink [65], change their optical properties when an electric current is applied [50]. For example, if applied to a transparent substrate, the display can switch between a transparent and a blue state. The color depends on the chemical composition of the ink, and can be tweaked such that a stack of EC-displays results in a full-color displays [83]. With no power applied in the colored state, the EC display takes several hours to return to its bleached state. As the substrate can be flexible, the technology is well-suited for wearable applications [108].

Thermochromic inks react to temperature changes with a change in color. By carefully mixing the dyes and precisely controlling temperature, several colors can be created on a single element [156]. Thermochromic displays are the slowest in our examples [34], especially when worn close to the body. Producing the heat necessary to change the display state requires time as fast temperature changes on the skin can lead to a sensation similar to touching a hot surface [166]. Cooling down to return to the initial state takes a variable amount of time depending on the environment and placement of the display. As a result, these displays can be used to render semi-persistent content, where slow refresh rates are not a concern.

Electrophoretic displays, also known as E Ink displays, consist of microcapsules filled with inversely charged black and white particles, which move up or down in the capsule when an electromagnetic field is applied [6]. The refresh rate of electrophoretic displays depends on the amount of content that needs to be updated, with an average refresh rate of around 10 frames per second [121]. While colored versions exists, these do not come on a flexible substrate, making them less suitable for wearable applications.

\section{WEARABLE DISPLAYS DESIGN SPACE}

Wearable displays are available in various forms and shapes, ranging from available, robust end-user products to fragile proof-of-concept prototypes. Several design spaces have been proposed to structure and analyze the work in this area $[135,136,160]$. Schneegass et al. proposed design spaces which consider various output and input modalities [136] and detailed implementation aspects such as screen resolution or their level of integration into the textile context [135]. Wang et al. [160] formulate a design space that integrates placement, form factor, interaction modality, and possible sensor signals. This design space was used to motivate a model for technology transfer and market readiness. These existing design spaces, however, have not been used to classify a number of existing projects.

As for on-body interaction, only the forearm is considered a suitable placement for both, touch input and visual output [123, 135]. We, therefore, argue that it is relevant to look at the output aspect 
individually. In this paper we focus on wearable displays, i.e. lightbased displays that are to be worn on the body. We deliberately did not consider shape changing displays (e.g., [107]) although most of the dimensions we defined are applicable to this type of display. The reason for this exclusion is that shape changing displays can both alter the visual appearance of the fabric or communicate with the user haptically by changing the tactile impression of the fabric. A complete picture would require including other haptic modalities such as temperature or vibration, which would go beyond the current scope of this survey. A detailed overview on shape changing displays can be found in the survey and design space by Rasmussen et al. [126].

Even though we restrict the scope as described above, the field of wearable displays covers a wide range of technologies, from high-fidelity smartwatches and AR-glasses to simple LEDs sewn into clothing or fitness bands. We, therefore, opted for a broader classification as this eases the creation of an overview and is more tolerant regarding unusual technologies or implementation. Our design space classifies wearable displays based on two overarching aspects:

(1) On-body placement: In contrast to common screen-based output, such as monitors, public displays, and smartphones, the placement of wearable displays greatly influences their physical properties, interactions afforded, and other requirements. Our design space categorises different types of wearable displays (e.g. clothing, accessory, on-body), and their location on the body to provide a better understanding (section 4).

(2) Display content: Visual attributes of the content widely vary among wearable displays. We categorise content based on technical properties of the display, such as temporal aspects and information density, and by the intended audience (section 5).

Figure 2 provides an overview of our resulting design space, with details regarding each of the included categories.

\section{Wearable Displays Database}

To populate our interactive design space with a variety of existing wearable displays, we used an approach similar to [137].

We started our search by performing a query for "wearable display" in the ACM digital library (dl.acm.org) and exporting the results to a BibTeX file which resulted in 2041 entries. We removed 108 publications containing the work "haptic", 48 containing "tactile", 8 containing "olfactory" and 9 "force"-displays, leaving 1815 publications in the database. We then cleared duplicates and filtered the collection for entries that contained both the work "wearable" and "display", which resulted in 144 entries.

To achieve a broader coverage, we performed a series of queries in DBPL (dblp.uni-trier.de). A search for "wearable display" returned 185 entries, "textile display" returned 19 entries, "on-skin display" returned 10 entries, "on-body display" returned 12 publications, while no entry was found for "body-worn display". As above, we removed entries for the words "haptic", "tactile", "olfactory", and "force" leading to a list of 150 publications.

After merging all results and clearing duplicate results, we started our classification process with 236 publications.
We downloaded the abstracts for these entries and removed those that did not fit the scope of our literature survey wich lies on collecting a large variety of wearable displays, ranging from prototypes to commercially available products. While head-mounted displays (HMDs), smart glasses and smart watches are wearable displays matching our definition, we did not include papers that presented a specific application or interaction technique for these, e.g., item selection, synchronization, and data sharing. We also did not include purely technical contributions that improve existing display types, e.g., that solely focus on power management or color reproduction.

For the remaining 79 entries, we downloaded the papers and classified them in our design space. While going through the papers, we found additional references that did not match our initial search terms, which we added to the corpus. The field of textile electronics is addressed not only by the research community, but also highly popular within the maker community. To get a complete coverage of existing systems, we included Instructables [66] as a data source, and explored all projects dealing with wearable displays. A search for "wearable display" on Thingiverse did not return any result. Finally, we completed the dataset by going through the proceedings of wearable and HCI themed conferences such as CHI, ISWC, UIST.

Overall, we accumulated a body of 129 wearable display systems which we classified along the dimensions of our design space (Figure 3).

\section{ON-BODY PLACEMENT}

The surface of the human body is quite unlike a desk, a wall, or any other flat planar surface where displays are typically placed. Wearable displays have exploited this property to present a wide range of possibilities and innovations. As such, there are three main techniques commonly used for placing wearable displays on the body:

(1) Accessories are items such as eyewear, headwear, watches \& bracelets, chains \& necklaces, handbags, belts \& straps, brooches, rings \& earrings, or shoes.

(2) Clothing can be sub-categorized into shirts or tops, jackets \& suits, notions (i.e., buttons and zippers), pants \& skirts, dresses, or underwear.

(3) Skin \& Body can also be used as surface onto which displays are directly applied.

For each of these, the exact location on the body can vary. As location influences reachability, the placement of a display on the body determines whether input can potentially be co-located with the display. Location also has a direct impact on the visibility [57] which affects its potential use for peripheral notifications. The location on the body also signals whether the displayed content is intended for wearers themselves, or whether it is a means of communication with the surrounding environment, or a combination of both. For each of the placement techniques, we further categorise placement of displays based on their location on the body.

\subsection{Accessories}

Accessories are typically worn as add-ons. They can serve aesthetic or cosmetic purposes, or can provide additional functionality. With 81 of the systems we surveyed, this is the largest category. 


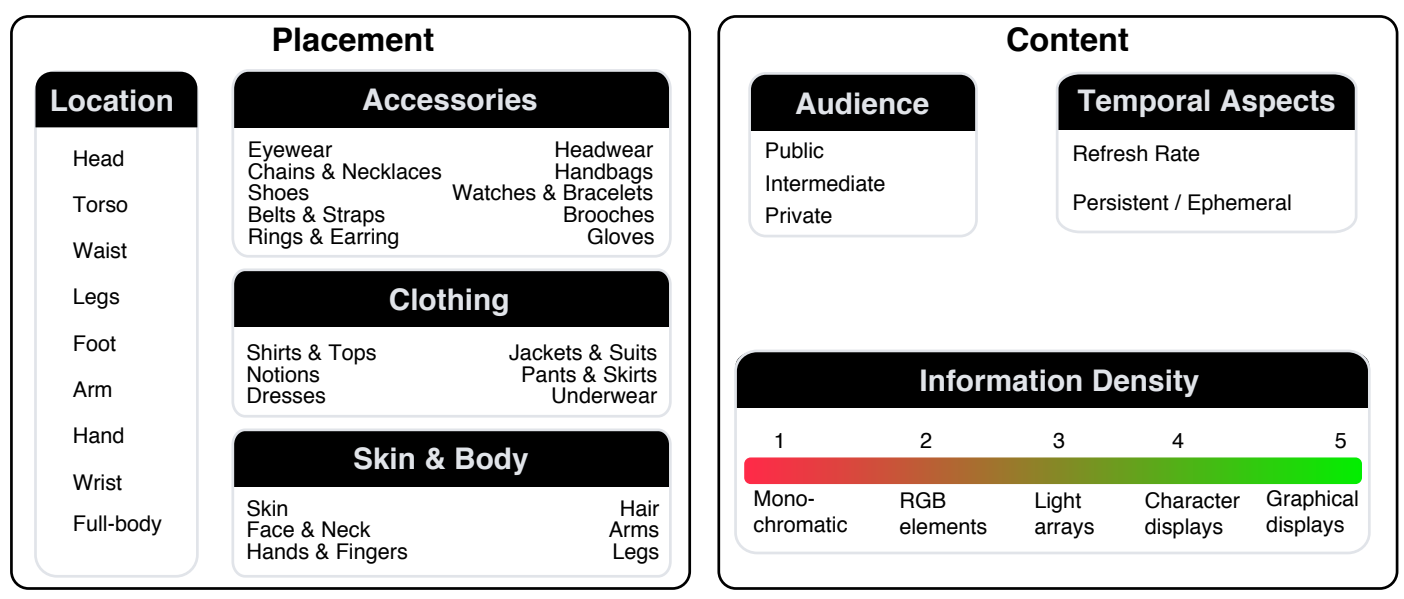

Figure 2: Our design space for wearable displays. A main factor for wearable displays is their placement on the body. In addition, they can be characterized by content properties, such as the intended audience, temporal features, and information density.

Eyewear. Placing a wearable display in the direct field of view ensures high visibility. A first implementation of the idea has been demonstrated in the late 1960s [146], and has undergone many iterations of miniaturization (see for example Steve Mann's EyeTap [100]) down to really wearable systems [104]. Smart glasses lack the capability of tracking their environment, thus basically just set a small screen right in front the eye which, through its proximity to the eye, results in a large coverage of the field of view $([37,49,111,138,172])$. Constant information display in direct sight, however, might be distracting and disturbing, and is not necessary for all purposes. Either the content on screen can be reduced to a specific subset relevant for the application at hand ([41, 113]), or abstracted and shown in the peripheral vision of the wearer $([23,28,95,113])$. In contrast to the previous example which are worn over long periods of time, Loupe [96] is a small viewfinderlike on-demand device that the user needs to place in front of the eye to use.

Headwear. As a personal information display, headwear is similar to eyewear in the sense that it adds visual information in the peripheral view of the user, but is mounted to a larger structure, e.g., a helmet. Early prototypes of AR glasses would also fall into this category, but only because of technical limitations as the vision behind these was always to create miniaturized display glasses. Similar to the glasses pushing the display into the wearers peripheral vision, LEaD [149] is a low-resolution visual indicator for motorcycle navigation which conveys the direction to take through a series of LEDs placed at the top of the visor.

Headwear is also ideal to communicate with the environment. Basic examples are helmets that add a brake light to (motor)cyclists' outfits $[12,94]$ The prototype by Walmink et al. [158] shows the rider's heart rate at the back of the bicycle helmet to inform other cyclists in a group about the well-being or fitness of the cyclist in front of them. In a more abstract sense, the helmet by Mann et al. visualizes the brain activity of its wearer [101]. A number of projects project content onto the wearer [140] or the environment $[48,59,106,133]$. The latter creates a larger visualization surface, but also makes the content inherently public. Displays can also be integrated into accessories like helmets [157], hats, caps [26], hoods, turbans, a hijab, or personal protective equipment [86]. Some displays can be used to adjust the visual appearance to the current outfit [33], or communicate information about the music the wearer is listening [71], social connection between wearers [68], current well-being [26], or the radiation the wearer is currently exposed to [63].

Watches \& Bracelets. The wrist is the most popular placement for wearable displays, as it is easy to read and to reach for interaction. Bracelets with a simple visualization are often used to increase the awareness for personal behavior, e.g., physical activity or drinking $[15,42-44]$ or in an inter-personal context as means for unobtrusive communication $[70,79,165]$ or group awareness $[2,165]$. On the commercial side, fitness trackers with a minimal visual interface like the Nike+ FuelBand [109] or the Fitbit Flex [40] were highly popular until smartwatches took included their functionality. The Tago Arc bracelet [85] builds around a flexible electrophoretic display and shows user-defined decorations fitting to the current outfit.

While popular smartwatches $[4,132]$ mostly contain a highresolution display, some concepts deliberately reduce the information density on the watch. Again, not all purposes require a high resolution [17]. Commercial products like the Withings Steel HR [168] only extend an analog watch with a minimal display for additional notifications. Building on the circular visualization pattern of the analog watch, a ring of LEDs can also serve as visualization for, e.g., environmental noise [142] or personal activity [171].

Even though modern smartwatches are equipped with highresolution displays, their screen real estate is still quite limited compared to, e.g., smartphones. LED displays around the watch can be useful extensions to ambiently show notifications while the main watch display is off $[10,122]$. The watch strap itself, while traditionally an inactive part of the watch, is an interesting component to augment with a display, as it allows to show content to the wearer independently of her arm's position [16]. This can be used to show private content to the wearer only, while the watch's main display 
is often visible for the environment [38]. With a display strap, the watch can either stay in place and the content on screen be adapted $[80,143]$ or include the rotation of the device around the arm as an additional metaphor for interaction [97]. The main watch display can also be extended in to a holographic representation, making it easier to follow, e.g., navigation instructions [163]. The wearable display can also be a companion to other wearable displays, either to be able to keep track of content outside the field-of-view an HMD can augment, or to make parts of an HMD view accessible to others [124].

A second approach it to use a pico-projector instead of a display that uses the lower arm as projection surface $[21,58,170]$ which creates a longitudinal interaction surface with a high resolution. While these systems could also be considered an augmentation of the lower arm, and thus be classified under Skin \& Body, LumiWatch [170] is clearly positioned as an interactive bracelet.

fewelry. Integrating electronics in jewelry is challenging as the space constraints are strick in order to keep it wearable [125].

Rings require the smallest footprint and therefore, communicate with the outside world using a single LED $[92,127]$. Chains \& Necklaces are not as restricted in their size, and can therefore increase the display size [110] or reach a higher information density [11, 72]. This can be used to show messages to the public with the intention to start conversations [11, 72, 105]. While not directly in the user's field-of-view, a necklace using projection technology can create a large visualization surface $[103,105]$.

Brooches are traditionally not flexible and, due to their size, not subject to wearability requirements such as breathability. This kind of accessory can, therefore, be created using traditional rigid electronics. Due to their placement on the torso, they are typically designed as a public display [39] or ornamental accessory [115].

Shoes. Displays integrated into shoes can serve an aesthetic purpose by adapting their visual appearance to the current outfit $[33,139]$. The Orphe One serves the special purpose of visually supporting the performance of a dancer by automatically changing colors [114]. The Wearable Aura [93] projects information around the user's feet based on individual connections that could serve as conversation starters.

Displays in shoes can also target the wearer itself, and show the overall physical activity level [73], or give information about a runner's current pace. [27]

Bags. Similar to the previous category, bags are also not subject to most wearability constraints, and even their flexibility can be limited without affecting the bag's functionality. The displays are meant for public audience, revealing information about the bag's content [25], environmental sounds [68], or online social identity [87].

Belts \& Straps. The forearm and the upper thighs provide a large support surface for interactive displays, which is commonly used by straps and belts. Simple ones are basically bargraphs that visualize heart rate [47] or the knee bending angle [3] for rehabilitation exercises. The forearm is a popular choice for larger screen real estate and additional interaction surface. This can either be for standalone use [29, 60, 112, 173], as collaborative interaction space for the wearer and another user [145], or a conjoint interaction space for a larger display [153]. Some straps also go around the hand [98], leaving more flexibility regarding placement than gloves.

Gloves. Gloves can offer a public display on the outside and a private display on the inside of the hand, but the flexibility requirements for the latter to not hinder the normal movement are high, making them fiction at this point [131]. The exposed placement of the hands make it suitable for safety illumination like a turn signal indicator [154].

\subsection{Clothing}

Clothing is a ubiquitous carrier for wearable technologies, as it is nearly always available and can be shaped around the wearable's function. However, it also poses hard requirements on the hardware as for a seamless integration, the additional components should have minimal influence on parameters such as wearability, fashionability, and breathability [46, 89]. These requirements limit the number of fully integrated wearable displays. Most of the systems are examples to introduce novices to the field of wearable computing by connecting LEDs to a battery holder through conductive thread. More complex examples are often build around the LilyPad [88], which is well-suited for prototyping, but also adds rigid elements to the supporting fabric. Despite these technical challenges, a series of interesting systems have been implemented and evaluated.

Shirts \& tops. Displays integrated into shirts \& Tops often communicate information about the wearer to its surroundings. This can be used to create a sense of connection between people wearing such shirts [120]. In a sports context, this can be health data [102] or game related stats, such as personal fouls in Basketball [117], or become part of the game action itself [19]. Such displays can also inform about the wearer's wellbeing [52, 98], exposure to noise [129] or polluted air [45] Two examples display content addressing the wearer, e.g., for navigation purposes [135] or as an ambient display to increase personal wellbeing $[52,54]$.

fackets \& Suits. As part of our clothing, jackets serve the purpose of providing a temporal protective layer against external influences, such as the weather. Therefore, they are well-suited for the integration of specialized functionality that is only relevant while wearing the jacket. These can be safety features such as turn indicators $[14,51]$, or light that turns on automatically in the dark [128] or in response to rain [22]. Other systems integrate (electrophoretic) displays into jackets \& suits for personal adaptation and expression $[20,35,128]$.

Buttons \& Zippers. Notions such as buttons and zippers are mostly of interest as means for reversible interconnection of wearable technology. Being exposed visual and tactile landmarks on the garment, however, also makes them an ideal subject for interactive features. Todi and Luyten proposed a design space of various input and output possibilities for buttons [148], including small OLED displays. Similarly, the 1-bit Textile electromagnetic display [84] could be applied, e.g., to buttons and their respective holes.

Skirts. Skirts provide a large support surface to integrate displays, but that space is usually only partially covered. Most project apply a series of tiles [156] or lines, e.g., to visualize where North is [134], 
environmental parameters $[5,67]$, or rotational speed while iceskating [53].

Dresses. Like their very nature, dresses and displays integrated into these have a strong emphasis on aesthetics. The display can be used to make the dress stand out through light and animation [81, 82, 159]. An integrated display can also make a dress more versatile and adapt it to various occasions by hiding or revealing, e.g, the back [69], or by changing its overall appearance [8, 36]. Some dresses also visualize information about the wearer, and interestingly about the wearer's wellbeing and stress level [130, 141], which reverses the protective aspect and emphasizes the expressive aspect of clothing.

\subsection{Skin \& Body}

From an implementation perspective, integrating displays into clothing is a reasonable choice, the fabric being an already existing support structure that is strong enough to carry the necessary electronics. The skin itself, however, has been a surface for personal expression through tattoos for centuries, which motivates a series of projects to add only a minimal layer of display technology directly on the human body.

Skintillates [91] extends temporary paper tattoos with conductive traces to power LEDs that become part of the ornament. A deeper integration of the display into aesthetics of the tattoo can be achieved using thermochromic $[76,77,161]$ or electroluminescent materials $[162,164]$ which can change the visual appearance of larger portions of the ornament. By using a projection onto the user's skin $[55,56,58]$, it is possible to create high-resolution visual output without the constrains of creating a flexible display

Displays based on biochemical reactions use chemicals that change their color depending on the amount of UV-radiation or $\mathrm{CO}_{2}$ it has been exposed to [75]. If applied subcutaneously, like a permanent tattoo, such chemicals can also react to the amount of certain elements in the wearer's body [151], such as Glucose and Sodium.

The human hair presents a large surface that can be used as output surface. Fire [150] and HäirIO [34] both use thermochromic ink to change the color of the hair while the latter also adds kinetics by adding shape-memory-alloys (SMA) to form a single active braid.

Adding a high-resolution display to the fingernails can be used to reduce the fat-finger problem by showing the content that is right below the finger $[144,167]$. Even with a limited information density the fingernail can be used as an unobtrusive output surface [35].

Hoang et al. explore the potential of using the entire body as a display surface, for example, to see through the skin and show the inner anatomy [64]. This kind of application can actually be useful in either the training or the performance of surgical procedures, even with a lower information density [62].

\section{DISPLAY CONTENT}

As evident from the above survey, there is a wide range of existing wearable displays, and they vary largely in terms of display properties, functions, and contents. The rendered content on wearable displays is generally determined by (1) the intended viewer(s), (2) temporal aspects of the display, and (3) information density. We discuss these in further detail here.

\subsection{Audience}

The aspect of intended audience is important to consider in the context of wearable displays. Depending on the placement, the display might be easily visible to others while showing sensitive content at the same time. The choice of placement, the choice of visualization, and the display technology are all influenced by the type of information that is to be shown by the display.

Some technologies can be used for all types of audiences, such as the tattoo-based $[75,91,151,162]$ displays that can either be in a publicly visible area such as the outer arm, but can also be placed at a mostly concealed position, e.g., the inner arm or the inner wrist, or an area that is mostly covered by clothing [112].

Public. Systems like the Orphe and ShiftWear sneakers [114, 139], the biking equipment[12, 14, 51, 94, 154, 158], the sport shirts $[102,117]$, the eyelid display [77], or the dresses [69, 81, 82, 130, 141, 159] are clearly meant for a public audience given their placement on the body. Other displays address the wearer, but are clearly visible for the public as, e.g., the visual content is projected onto the environment [55, 56, 87, 103, 105, 106, 133].

The same device can also show public and private content simultaneously if some areas of the hardware are concealed by their position $[16,97]$ or by special technology such as retroreflective material [145] or lenticular lenses.

Intermediate. If personal information is to be communicated through a display that is publicly visible because it is physically exposed, e.g., as a ring on the finger [127] or on the shoes [27], an adequate mapping can conceal the meaning of the message. The information is then only accessible to those knowing the mapping $[38,79]$.

An interesting aspect in this category is, whether the public can infer the meaning behind the display, or if only the wearer has the contextual knowledge to interpret the message. For systems that quickly react on public parameters, e.g., environmental noise [67, $68,129,142]$ or imminent actions of the wearer $[27,53,134]$, the mapping might be easy to grasp for an observer.

Generally, every public display can be turned into an intermediate one by applying a concealing mapping to the information shown, as exemplified in [38].

Private. Private displays are designed to show their content to the wearer only, which in our survey, applies to all of the eyewear $[23,28,37,41,49,95,96,99,111,113,146]$ and some of the headwear [149]. A publicly visible display can, if applicable from the form factor, show sensitive information by integrating a mechanism that only shows content when the display is facing the wearer $[16,97,143]$.

\subsection{Temporal Aspects}

The various technologies also differ in two temporal aspects: the time it takes to update content on the display, and the persistence of the content on the display whenever power is cut. If we take wearable displays that primarily serve the context of adjusting the optical appearance of the garment when dressing, e.g., to match other elements, the refresh rate is nearly irrelevant. In this scenario, having a bi-stable, persistent display would dramatically reduce power consumption and further reduce the amount of hardware to 


\begin{aligned} Technology & Share \\ \hline LED & $34 \% \\$ LCD & $17 \% \\$ Projection & $12 \% \\$ Thermochromic & $12 \% \\$ Electrophoretic & $11 \% \\$ Electroluminescent & $6 \% \\$ OLED & $4 \% \\$ Electrochromic & $3 \% \\$ Biochemical & $1 \% \\$ CRT & $1 \%\end{aligned}$

Table 1: The various display technologies and how often these are used in wearable displays.

be integrated [33]. Overall, a low power consumption is favorable in a wearable context as batteries become increasingly unwearable with size due to their weight and shape.

LEDs are the fastest with reaction times in the order of nanoseconds and the most popular technology from our survey but they require constant power supply to be illuminated. Using them in a persistence of vision (POV) display makes the information ephemeral in even two ways as it is additionally dependent on the wearer's movement [90]. Electroluminescent displays have similar characteristics, but require additional hardware to create the high-voltage high-frequency AC field. LCDs are fast and low-power, but require a backlight in order to be visible under all conditions.

OLED coating might play a large role in the future, as it is light emitting, low power, and only has minimal effect on wearability, but this technology is still in an early stage [152].

Thermochromic displays [32, 34, 47, 52, 68, 71, 76, 77, 120, 141, $150,156,161]$ are the slowest in our comparison, especially when worn close to the body. Cooling down to return to the initial state takes a variable amount of time depending on the environment and placement of the display, i.e., the amount of heat that can be applied or dissipate from the display. Producing the heat necessary to change the display state requires time as fast temperature changes on the skin can lead to a sensation similar to touching a hot surface [166]. The transition temperature of the pigments is only slightly below body temperature [34], which means that cooling down is slower than heating it up. As a result, these displays can be used to render semi-persistent content, where slow refresh rates are not a concern.

Electrochromic displays $[69,70,73,129]$ require a noticeable amount of time to change their state [108]. Their working principle makes them a capacitor, which means that once they are charged to reach their activated state, they will discharge over time and eventually return to their inactive state, but this can take a considerable amount of time. For example, Ynvisible [65] specifies a persistence in the active state of "several hours".

Electrophoretic (E-ink) displays [16, 20, 33, 35, 60, 69, 73, 80, $84,85,115,129,139,143,147,153]$ are truly bi-stable and only require power when the display content is updated. This means that the screen content remains unchanged, even if the display is disconnected from the power source. The update rate depends on the amount of content that needs to be updated, and can range from
$0.1 s$ to $1 s$ [121]. Overall, only $11 \%$ of the 129 projects we evaluated use persistent displays.

\subsection{Information Density}

Wearable displays cover a wide range of information that these can potentially display: Some only consist of a few single LEDs while others can show high-resolution graphical content. We quantified the information density on a five-point scale. The first (lowest) level contains displays that only have a low number $(\leq 5)$ of light elements that only switch between two states, i.e., single LEDs or a number of LEDs that light up simultaneously. Elements do not need to be point light sources, but can also be larger electroluminescent areas [22], or LEDs attached to optical fibers [9]. The second contains projects that still have a limited number of elements, but which can display a larger number of states, e.g., RGB LEDs. At this point, it is important to note that users might not be able to perceive the same number of different states that the display is technically able to present [17]. The third level is for larger arrays which can visualize single digits, icons, or running text. The fourth is for LCD which can show entire phrases, but no full graphical content. The highest level of information density is provided by full graphical displays.

\section{INTERACTIVE DESIGN SPACE EXPLORATION}

The web-based tool to explore the data is available at https:// florianheller.github.io/displaydesignspace and is updated automatically whenever new data is committed to the repository. It allows to filter and visualize the projects in the database along various dimensions in order to find the elements of interest.

The data underlying this analysis is publicly available on Github ${ }^{1}$ and consists of a simple JSON-formatted file. In extension to our design space, this database contains additional information such as a link to the original publication, author information, the year of publication, and the type of display technology that is used. Given the wide variety of sources for wearable display prototypes, aggregating the data is challenging and we acknowledge that our project corpus is limited. We invite fellow researchers to contribute to this explorable design space to complete it and keep it up-todate with future publications and projects. Github features such as the issue tracker and pull requests can also serve as a discussion platform in case a certain classification is challenged.

\subsection{Use Cases}

Given the existing classification and taking into account possible future additions, we envision a number of use-cases for the webbased tool and the raw underlying data.

6.1.1 Finding Examples for Specific Use-Cases. As wearable displays become more available, interface designers are evaluating the use of additional interaction surfaces, for example in a professional context [61]. The explorable design space allows the designer to quickly search for examples of wearables that are placed at the designated position and, ideally, matching the set requirements

\footnotetext{
${ }^{1}$ https://github.com/florianheller/displaydesignspace
} 


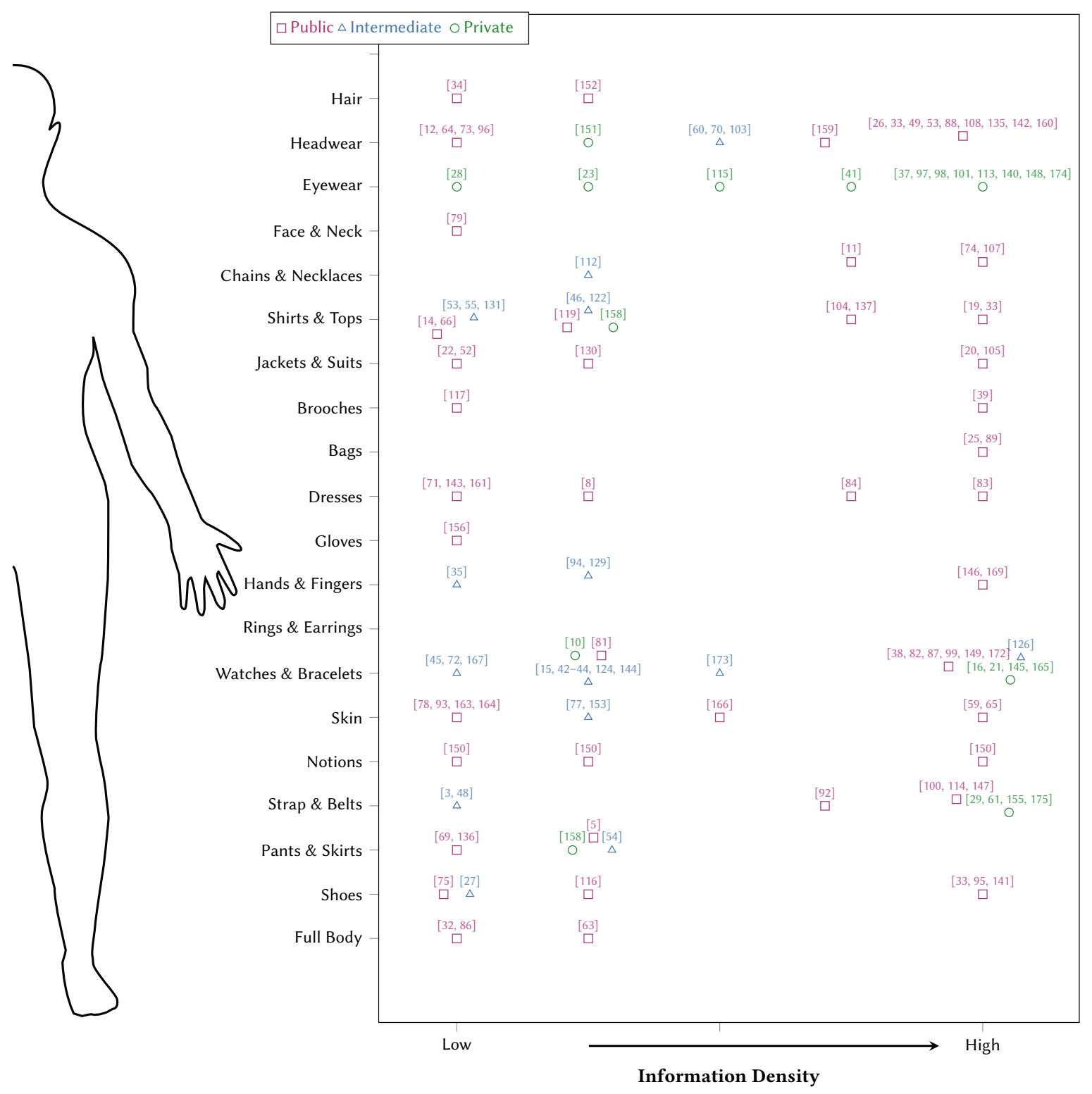

Figure 3: Wearable displays from literature classified into our design space. The amount of information shown on a display increases from left (low or abstract) to right (high or detailed). We refer to the interactive design space tool to explore the projects in more detail.

regarding, e.g., refresh-rate or information density. If the search results report results of a formal evaluation, the decision can be based on solid grounds without having to create own initial prototypes.

6.1.2 Simulation of Wearable Displays. In an industrial context, more and more production environments get simulated in VR [74]. This enables designers to evaluate and improve things such as ergonomics, or production flow. Similar to the use-case outlined above, we envision our dataset to be a useful resource for such simulations by automatically creating virtual counterparts of wearable displays. Attached to a virtual human body, parameters such as placement, visibility, and reachability can be evaluated in-situ. With the information currently included in the dataset, the generic 3D models for visualization have to be rendered based on the categories from the design-space at the expense of correct reproduction of display size, resolution, and position. Therefore, we suggest the inclusion of a few key characteristics in future publications on wearable displays, as described in subsection 6.2. 


\subsection{Future Directions}

Based on the survey, we outline a few directions for future development in the area of wearable displays.

6.2.1 Higher Integration into Textile Materials. The materials we wear, be they traditional or high-tech, generally aim at being breathable and letting moisture escape. Many of the displays however, especially those with a high information density, are closed surfaces. Wearable displays with individual miniaturized LEDs and interconnected using conductive thread [7] significantly minimize this problem. With these point light sources, however, it is necessary to use a diffuser layer to evenly light up a larger area [8]. Electroluminescent coatings on fabric base-layers bring the display even closer to a textile nature [30], but they only support a very limited number of colors, and their wiring gets increasingly complex with an increasing number of separate light emitting areas, meaning they are mostly suited for larger unicolor surfaces.

6.2.2 Increase Cultural Diversity. Clothing is highly individual and diverse, yet the proposed systems largely address a casual western dressing style. We would like to see more examples of appropriation to wearable displays to the local clothing styles across the world. For example, the Breaking of Dawn Jacket [128] builds on the traditional looks and material of Scandinavian and Arctic countries and proposes an application specific to the long periods without sunlight encountered in these countries.

6.2.3 Automatic Content Adaptation. The variation between the displays we surveyed is already large. As of now, the displays only show content that was specifically tailored to their characteristics. If we look at popular wearable eco-systems, however, we see that multi-purpose devices such as smart phones and watches that run third-party Apps outnumber those who are not part of such ecosystem. If we now imagine future developers having to adapt their app to the multitude of wearable displays, the complexity becomes unmanageable. Based on our classification, a developer can derive which display categories are essential to cover. Furthermore, we imagine a software tool that automatically adapts the graphical representation of an App to the specific display at hand. The information for this adaptation, again, comes from our classification and allows the tool decide which assets to request from the developer. For example, if a central part of the App is showing a percentage, the software would ask for a visualization optimized for, e.g., a high-resolution color display, a small, low resolution display, and a single RGB-LED. If the developer provides these, the adaptation layer can then generate the remaining variations.

6.2.4 Detailed Information. To support quick categorization of wearable displays, future publications should contain some specific information on the hardware. For the two use-cases outlined above to produce optimal results, future publications on wearable displays should include specific information on the dimensions of the display surface and the enclosure. Furthermore, the pixel resolution, the refresh rate, and the color depth should be reported. While these characteristics are essentially how we would characterize a desktop display, when going into the mobile domain the issue of power consumption becomes relevant, and based on that whether the screen content is persistent or ephemeral. Moving further to the wearable domain, features such as breathability and flexibility are worth reporting, in order to differentiate between displays that are basically a sheet of plastic placed on clothing, or wether the display is a more integral part of it allowing air to pass the display surface. Even though research prototypes are often different from the envisioned device they represent, information on the intended dimensions and characteristics will allow a more precise simulation, or a faster decision for or against a certain concept.

6.2.5 Applications for a Multi-Device Ecology. If we think of a dense body area network with a multitude of displays, visual output can or has to be distributed across a variety of displays. PortalWare [124] already sketches some possibilities where two wearable displays complement each other. In conjunction with the automatic content adaptation outlined above, an application could split the output onto multiple displays as in [118] and thus, does not require one single display that covers all the application's requirements.

\section{CONCLUSION}

Wearable displays have found several applications in recent times, and enable new forms of ubiquitous interactions. The space of wearable displays is large and fragmented-there are several types of displays, varying in function, capabilities, and intended audience (cf. Figure 2). It can be a challenge to design content appropriate for different displays, especially as the space grows rapidly.

In this paper, we surveyed available wearable displays, from research, industry, and maker communities. We defined a design space based on body location, type, viewing audience, and temporal capabilities of the displays, and classified 129 items along these dimensions. The result of this work find application in a web-based tool that interactively presents prior and current works. We suggest four main benefits our work can provide to researchers, designers, and practitioners.

First, by providing a broad overview, it can serve as an introduction to the wide-spanning area of wearable displays. Manual literature search can be cumbersome and intimidating since works in this area are spread across different domains. Due to this, there is a risk that the search is limited to a local domain, and knowledge is not shared across the field.

Second, through filtering and refinement, it can support focused investigation into sub-spaces. This is particularly useful to inform design choices for a new technology or interaction technique, and can avoid replication of effort.

Third, by highlighting gaps and blind spots, it can enable detection of new possibilities for novel wearable displays. Through transfer of knowledge, researchers and designers could quickly spot opportunities to contribute towards filling in, or expanding the design space.

Finally, our platform can be used as a tool for contemplation, and guide designers when thinking about how content should, or could, be adapted to different displays. In a likely future setting, users might possess a plurality of wearable displays, and could chose to adorn subsets of them, at given times, in different configurations. Under such circumstances, it would be necessary to customise the content to suit a user's given setting. Our work lays the foundation to explore (semi-)automatic adaptation of content, such that an appropriate visual representation is chosen based on qualities of 
the devices used, the user, and the context of use. We aim to build upon this work further by developing techniques to optimise and distribute contents to different wearable displays automatically.

\section{OPEN CODE}

The web tool (https://florianheller.github.io/displaydesignspace ) and data repository (https://github.com/florianheller/displaydesignspace) are fully open-sourced, and available. To support evolution of the design space, and to keep it up-to-date, we invite contributions from the community. Complete documentation and instructions, are available on the GitHub project page.

\section{ACKNOWLEDGMENTS}

This work was partly funded by the Department of Communications and Networking (Comnet, Aalto University), and Academy of Finland projects 'Human Automata' and 'BAD'. This research was partially funded by Flanders Make, the strategic research centre for the manufacturing industry, through its projects 'SmartHandler' and 'Ergo-EyeHand'.

\section{REFERENCES}

[1] Adafruit. 2012. Flora - Wearable Electronics Platform. https://www.adafruit com/flora.

[2] Petra Ahde and Jussi Mikkonen. 2008. Hello: Bracelets Communicating Nearby Presence of Friends. In Proceedings of the Tenth Anniversary Conference on Participatory Design 2008 (Bloomington, Indiana) (PDC '08). Indiana University, USA, 324-325.

[3] Swamy Ananthanarayan, Miranda Sheh, Alice Chien, Halley Profita, and Katie Siek. 2013. Pt Viz: Towards a Wearable Device for Visualizing Knee Rehabilitation Exercises. In Proceedings of the SIGCHI Conference on Human Factors in Computing Systems (Paris, France) (CHI '13). ACM, New York, NY, USA 1247-1250. https://doi.org/10.1145/2470654.2466161

[4] Apple. 2015. Watch. https://www.apple.com/watch/.

[5] Rain Ashford. 2014. Baroesque Barometric Skirt. In Proceedings of the 2014 ACM International Symposium on Wearable Computers: Adjunct Program (Seattle, Washington) (ISWC '14 Adjunct). ACM, New York, NY, USA, 9-14. https: //doi.org/10.1145/2641248.2641271

[6] Peng Fei Bai, Robert Andrew Hayes, Mingliang Jin, Lingling Shui, Zi Chuan Yi, Li Wang, Xiao Zhang, and Guofu Zhou. 2014. Review of paper-like display technologies. Progress in electromagnetics research 147 (2014), 95-116.

[7] Mary Ellen Berglund, Julia Duvall, Cory Simon, and Lucy E. Dunne. 2015 Surface-Mount Component Attachment for e-Textiles. In Proceedings of the 2015 ACM International Symposium on Wearable Computers (Osaka, Japan) (ISWC '15). ACM, New York, NY, USA, 65-66. https://doi.org/10.1145/2802083.2808413

[8] Mary Ellen Berglund, Esther Foo, Md. Tahmidul Islam Molla, Smitha Muthya Sudheendra, Crystal Compton, and Lucy E. Dunne. 2018. MAKE IT BLUE: A Controllable, Color-Changing Dynamic Costume. In Proceedings of the 2018 ACM International Symposium on Wearable Computers (Singapore, Singapore) (ISWC '18). ACM, New York, NY, USA, 236-241. https://doi.org/10.1145/3267242. 3267304

[9] Joanna Berzowska and Maksim Skorobogatiy. 2009. Karma Chameleon Jacquard-Woven Photonic Fiber Display. In SIGGRAPH 2009: Talks (New Orleans, Louisiana) (SIGGRAPH '09). ACM, New York, NY, USA, Article 11, 1 pages. https://doi.org/10.1145/1597990.1598001

[10] Gabor Blasko, Chandra Narayanaswami, and Steven Feiner. 2006. Prototyp ing Retractable String-based Interaction Techniques for Dual-display Mobile Devices. In Proceedings of the SIGCHI Conference on Human Factors in Comput ing Systems (Montréal, Québec, Canada) (CHI '06). ACM, New York, NY, USA, 369-372. https://doi.org/10.1145/1124772.1124827

[11] Richard Borovoy, Fred Martin, Sunil Vemuri, Mitchel Resnick, Brian Silverman, and Chris Hancock. 1998. Meme Tags and Community Mirrors: Moving from Conferences to Collaboration. In Proceedings of the 1998 ACM Conference on Computer Supported Cooperative Work (Seattle, Washington, USA) (CSCW '98). ACM, New York, NY, USA, 159-168. https://doi.org/10.1145/289444.289490

[12] BrakeFree 2017. Brake Free - The Smart Brake Light for Motorcyclists. http: //www.brakefreetech.com.

[13] Frederik Brudy, Christian Holz, Roman Rädle, Chi-Jui Wu, Steven Houben, Clemens Nylandsted Klokmose, and Nicolai Marquardt. 2019. Cross-Device Taxonomy: Survey, Opportunities and Challenges of Interactions Spanning
Across Multiple Devices. In Proceedings of the 2019 CHI Conference on Human Factors in Computing Systems (Glasgow, Scotland Uk) (CHI '19). ACM, New York, NY, USA, 1-28. https://doi.org/10.1145/3290605.3300792

[14] Leah Buechley. 2008. Turn Signal Biking Jacket. Instructables. http://www. instructables.com/id/turn-signal-biking-jacket/.

[15] Patrick Burns, Christopher Lueg, and Shlomo Berkovsky. 2012. Activmon: Encouraging Physical Activity Through Ambient Social Awareness. In Extended Abstracts on Human Factors in Computing Systems (Austin, Texas, USA) (CHI EA '12). ACM, New York, NY, USA, 2363-2368. https://doi.org/10.1145/2212776. 2223803

[16] Jesse Burstyn, Paul Strohmeier, and Roel Vertegaal. 2015. DisplaySkin: Exploring Pose-Aware Displays on a Flexible Electrophoretic Wristband. In Proceedings of the Ninth International Conference on Tangible, Embedded, and Embodied Interaction (Stanford, California, USA) (TEI '15). ACM, New York, NY, USA, 165-172. https://doi.org/10.1145/2677199.2680596

[17] Christopher Campbell and Peter Tarasewich. 2004. What Can You Say with Only Three Pixels?. In Architecture of Computing Systems - ARCS 2014. Springer, Berlin, Heidelberg, 1-12. https://doi.org/10.1007/b100594

[18] Stuart K. Card, Jock D. Mackinlay, and George G. Robertson. 1991. A Morphological Analysis of the Design Space of Input Devices. ACM Trans. Inf. Syst. 9, 2 (April 1991), 99-122. https://doi.org/10.1145/123078.128726

[19] Sylvia H. Cheng, Kibum Kim, and Roel Vertegaal. 2011. TagURit: a proximitybased game of tag using lumalive e-textile displays. In Proceedings of the International Conference on Human Factors in Computing Systems, CHI 2011, Extended Abstracts Volume, Vancouver, BC, Canada, May 7-12, 2011. ACM, New York, NY, USA, 1147-1152. https://doi.org/10.1145/1979742.1979707

[20] Bianca Cheng Costanzo. 2016. MAREA. http://cargocollective.com/bia/MAREA.

[21] Cicret 2018. Cicret Bracelet. https://www.cicret.com.

[22] Elise Co and Nikita Pashenkov. 2008. Emerging Display Technologies for Organic User Interfaces. Commun. ACM 51, 6 (June 2008), 45-47. https: //doi.org/10.1145/1349026.1349036

[23] Vanessa Cobus, Hannah Meyer, Swamy Ananthanarayan, Susanne Boll, and Wilko Heuten. 2018. Towards Reducing Alarm Fatigue: Peripheral Light Pattern Design for Critical Care Alarms. In Proceedings of the 10th Nordic Conference on Human-Computer Interaction (Oslo, Norway) (NordiCHI '18). ACM, New York, NY, USA, 654-663. https://doi.org/10.1145/3240167.3240218

[24] Zane Cochran, Clint Zeagler, and Sonia McCall. 2015. Addressing Dresses: User Interface Allowing for Interdisciplinary Design and Calibration of LED Embedded Garments. In Proceedings of the 2015 ACM International Symposium on Wearable Computers (Osaka, Japan) (ISWC '15). ACM, New York, NY, USA, 61-64. https://doi.org/10.1145/2802083.2808403

[25] Ashley Colley, Minna Pakanen, Saara Koskinen, Kirsi Mikkonen, and Jonna Häkkilä. 2016. Smart Handbag As a Wearable Public Display - Exploring Concepts and User Perceptions. In Proceedings of the 7th Augmented Human International Conference (Geneva, Switzerland) (AH '16). ACM, New York, NY, USA, 7:1-7:8. https://doi.org/10.1145/2875194.2875212

[26] Ashley Colley, Bastian Pfleging, Florian Alt, and Jonna Häkkilä. 2020. Exploring public wearable display of wellness tracker data. Int. 7. Hum. Comput. Stud. 138 (2020), 102408. https://doi.org/10.1016/j.ijhcs.2020.102408

[27] Ashley Colley, Paweł W. Woźniak, Francisco Kiss, and Jonna Häkkilä. 2018. Shoe Integrated Displays: A Prototype Sports Shoe Display and Design Space. In Proceedings of the 10th Nordic Conference on Human-Computer Interaction (Oslo, Norway) (NordiCHI '18). ACM, New York, NY, USA, 39-46. https://doi. org $/ 10.1145 / 3240167.3240216$

[28] Enrico Costanza, Samuel A. Inverso, Elan Pavlov, Rebecca Allen, and Pattie Maes. 2006. Eye-q: Eyeglass Peripheral Display for Subtle Intimate Notifications. In Proceedings of the 8th Conference on Human-Computer Interaction with Mobile Devices and Services (Helsinki, Finland) (MobileHCI '06). ACM, New York, NY, USA, 211-218. https://doi.org/10.1145/1152215.1152261

[29] Alexandru Dancu, Mickaël Fourgeaud, Mohammad Obaid, Morten Fjeld, and Niklas Elmqvist. 2015. Map Navigation Using a Wearable Mid-air Display. In Proceedings of the 17th International Conference on Human-Computer Interaction with Mobile Devices and Services (Copenhagen, Denmark) (MobileHCI '15). ACM, New York, NY, USA, 71-76. https://doi.org/10.1145/2785830.2785876

[30] Marc de Vos, Russel Torah, Steve Beeby, and John Tudor. 2014. Functional Electronic Screen-printing - Electroluminescent Lamps on Fabric. Procedia Engineering 87 (2014), 1513 - 1516. https://doi.org/10.1016/j.proeng.2014.11.586 EUROSENSORS 2014, the 28th European Conference on Solid-State Transducers.

[31] M. de Vos, R. Torah, M. Glanc-Gostkiewicz, and J. Tudor. 2016. A Complex Multilayer Screen-Printed Electroluminescent Watch Display on Fabric. Fournal of Display Technology 12, 12 (Dec 2016), 1757-1763. https://doi.org/10.1109/ JDT.2016.2613906

[32] Laura Devendorf, Joanne Lo, Noura Howell, Jung Lin Lee, Nan-Wei Gong, M. Emre Karagozler, Shiho Fukuhara, Ivan Poupyrev, Eric Paulos, and Kimiko Ryokai. 2016. "I Don't Want to Wear a Screen": Probing Perceptions of and Possibilities for Dynamic Displays on Clothing. In Proceedings of the 2016 CHI Conference on Human Factors in Computing Systems (San Jose, California, USA) (CHI '16). ACM, New York, NY, USA, 6028-6039. https://doi.org/10.1145/2858036.2858192 
[33] Christine Dierk, Molly Jane Pearce Nicholas, and Eric Paulos. 2018. AlterWear: Battery-Free Wearable Displays for Opportunistic Interactions. In Proceedings of the 2018 CHI Conference on Human Factors in Computing Systems (Montreal QC, Canada) (CHI '18). ACM, New York, NY, USA, 220:1-220:11. https://doi. org/10.1145/3173574.3173794

[34] Christine Dierk, Sarah Sterman, Molly Jane Pearce Nicholas, and Eric Paulos. 2018. HäirIÖ: Human Hair As Interactive Material. In Proceedings of the Twelfth International Conference on Tangible, Embedded, and Embodied Interaction (TEI '18). ACM, New York, NY, USA, 148-157. https://doi.org/10.1145/ 3173225.3173232

[35] Christine Dierk, Tomás Vega Gálvez, and Eric Paulos. 2017. AlterNail: Ambient, Batteryless, Stateful, Dynamic Displays at Your Fingertips. In Proceedings of the 2017 CHI Conference on Human Factors in Computing Systems (CHI '17). ACM New York, NY, USA, 6754-6759. https://doi.org/10.1145/3025453.3025924

[36] DNP 2018. DNP - Dai Nippon Printing. http://www.dnp.co.jp.

[37] Epson. 2016. Moverio Smart Glasses. https://moverio.epson.com/.

[38] Noelene Fajardo and Andrew Vande Moere. 2008. ExternalEyes: Evaluating the Visual Abstraction of Human Emotion on a Public Wearable Display Device. In Proceedings of the 20th Australasian Conference on Computer-Human Interaction: Designing for Habitus and Habitat (Cairns, Australia) (OZCHI '08). ACM, New York, NY, USA, 247-250. https://doi.org/10.1145/1517744.1517762

[39] Jennica Falk and Staffan Björk. 1999. The BubbleBadge: A Wearable Public Display. In CHI '99 Extended Abstracts on Human Factors in Computing Systems (Pittsburgh, Pennsylvania) (CHI EA '99). ACM, New York, NY, USA, 318-319. https://doi.org/10.1145/632716.632909

[40] Fitbit. 2017. Flex2. https://www.fitbit.com/au/flex2

[41] Form. 2019. Form Swim Goggles With a Smart Display. https://www.formswim. com/.

[42] Jutta Fortmann, Vanessa Cobus, Wilko Heuten, and Susanne Boll. 2014. WaterJewel: Design and Evaluation of a Bracelet to Promote a Better Drinking Behaviour. In Proceedings of the 13th International Conference on Mobile and Ubiquitous Multimedia (Melbourne, Victoria, Australia) (MUM '14). ACM, New York, NY, USA, 58-67. https://doi.org/10.1145/2677972.2677976

[43] Jutta Fortmann, Heiko Müller, Susanne Boll, and Wilko Heuten. 2013. Illumee: Aesthetic Light Bracelet As a Wearable Information Display for Everyday Life. In Proceedings of the 2013 ACM Conference on Pervasive and Ubiquitous Computing Adjunct Publication (UbiComp '13 Adjunct). ACM, New York, NY, USA, 393-396. https://doi.org/10.1145/2494091.2495970

[44] Jutta Fortmann, Janko Timmermann, Bengt Lüers, Marius Wybrands, Wilko Heuten, and Susanne Boll. 2015. LightWatch: A Wearable Light Display for Personal Exertion. In Proceedings of the 15th IFIP TC 13 International Conference on Human-Computer Interaction (INTERACT '15, Vol. 9299). Springer, Berlin, Germany, 582-585. https://doi.org/10.1007/978-3-319-22723-8_66

[45] Mary Gao. 2019. EqualAir: Wearable NeoPixel Display Triggered by Air Pollution Sensor. Instructables. https://www.instructables.com/id/EqualAirWearable-NeoPixel-Display-Triggered-by-Ai/.

[46] F Gemperle, C Kasabach, J Stivoric, Malcolm Bauer, and R Martin. 1998. Design for Wearability. In Proceedings of the 2nd IEEE International Symposium on Wearable Computers (ISWC '98). IEEE, New York, NY, USA, 116-122. https: //doi.org/10.1109/ISWC.1998.729537

[47] Çağlar Genç, Yavuz Ali Ekmekçioğlu, Fuat Balci, Hakan Ūrey, and Oguzhan Ozcan. 2019. Howel: A Soft Wearable with Dynamic Textile Patterns As an Ambient Display for Cardio Training. In Extended Abstracts of the 2019 CHI Conference on Human Factors in Computing Systems (Glasgow, Scotland Uk) (CHI EA '19). ACM, New York, NY, USA, LBW1312:1-LBW1312:6. https://doi. org/10.1145/3290607.3312857

[48] Çağlar Genç, Shoaib Soomro, Yalçın Duyan, Selim Ölçer, Fuat Balcı, Hakan Ürey, and Oğuzhan Özcan. 2016. Head Mounted Projection Display \& Visual Attention Visual Attentional Processing of Head Referenced Static and Dynamic Displays While in Motion and Standing. In Proceedings of the 2016 CHI Conference on Human Factors in Computing Systems (San Jose, California, USA) (CHI '16). ACM, New York, NY, USA, 1538-1547. https://doi.org/10.1145/2858036.2858449

[49] Google. 2013. Glass. https://x.company/glass/.

[50] Claes-Göran Granqvist. 2015. Electrochromic Metal Oxides: An Introduction to Materials and Devices. John Wiley \& Sons, Ltd, Chapter 1, 1-40. https://doi.org/10.1002/9783527679850.ch1 arXiv:https://onlinelibrary.wiley.com/doi/pdf/10.1002/9783527679850.ch1

[51] Tobias Grosse-Puppendahl, Oskar Bechtold, Lukas Strassel, David Jakob, Andreas Braun, and Arjan Kuijper. 2015. Enhancing Traffic Safety with Wearable Low-resolution Displays. In Proceedings of the 2Nd International Workshop on Sensor-based Activity Recognition and Interaction (Rostock, Germany) (iWOAR '15). ACM, New York, NY, USA, 10:1-10:10. https://doi.org/10.1145/2790044 2790059

[52] Jonna Häkkilä, Ashley Colley, Paula Roinesalo, Tuomas Lappalainen, Inka Rantala, and Jani Väyrynen. 2017. Wearable augmented reality display for wellness. In Proceedings of the 6th ACM International Symposium on Pervasive Displays (PerDis '17), Marc Langheinrich and Sarah Clinch (Eds.). ACM, New
York, NY, USA, 25:1-25:2. https://doi.org/10.1145/3078810.3084348

[53] Jonna Häkkilä, Veera Helander, Diana Jamoido, and Ashley Colley. 2018. Designing an Interactive Ice Skating Dress for Young Athletes. In Proceedings of the 2018 ACM International foint Conference and 2018 International Symposium on Pervasive and Ubiquitous Computing and Wearable Computers (Singapore, Singapore) (UbiComp '18). ACM, New York, NY, USA, 734-737. https: //doi.org/10.1145/3267305.3267702

[54] Emmi Harjuniemi, Ashley Colley, Piia Rytilahti, Hong Li, Jesse Forest, and Jonna Häkkilä. 2018. Idle Stripes Shirt: Ambient Wearable Display for Activity Tracking. In Proceedings of the 2018 ACM International Symposium on Wearable Computers (Singapore, Singapore) (ISWC '18). ACM, New York, NY, USA, 254259. https://doi.org/10.1145/3267242.3267303

[55] Chris Harrison, Hrvoje Benko, and Andrew D. Wilson. 2011. OmniTouch: Wearable Multitouch Interaction Everywhere. In Proceedings of the 24th Annual ACM Symposium on User Interface Software and Technology (Santa Barbara, California, USA) (UIST '11). ACM, New York, NY, USA, 441-450. https://doi. org $/ 10.1145 / 2047196.2047255$

[56] Chris Harrison and Haakon Faste. 2014. Implications of Location and Touch for On-Body Projected Interfaces. In Proceedings of the 2014 Conference on Designing Interactive Systems (Vancouver, BC, Canada) (DIS '14). ACM, New York, NY, USA, 543-552. https://doi.org/10.1145/2598510.2598587

[57] Chris Harrison, Brian Y Lim, Aubrey Shick, and Scott E Hudson. 2009. Where to Locate Wearable Displays?: Reaction Time Performance of Visual Alerts from Tip to Toe. In Proceedings of the SIGCHI Conference on Human Factors in Computing Systems (CHI '09). ACM, New York, NY, USA, 941-944. https: //doi.org/10.1145/1518701.1518845

[58] Chris Harrison, Desney Tan, and Dan Morris. 2010. Skinput: Appropriating the Body As an Input Surface. In Proceedings of the SIGCHI Conference on Human Factors in Computing Systems (Atlanta, Georgia, USA) (CHI '10). ACM, New York, NY, USA, 453-462. https://doi.org/10.1145/1753326.1753394

[59] Jeremy Hartmann, Yen-Ting Yeh, and Daniel Vogel. 2020. AAR: Augmenting a Wearable Augmented Reality Display with an Actuated Head-Mounted Projector. In UIST '20: The 33rd Annual ACM Symposium on User Interface Software and Technology, Virtual Event, USA, October 20-23, 2020, Shamsi T. Iqbal, Karon E. MacLean, Fanny Chevalier, and Stefanie Mueller (Eds.). ACM, New York, NY, USA, 445-458. https://doi.org/10.1145/3379337.3415849

[60] Florian Heller and Kris Luyten. 2019. TaskHerder: A Wearable Minimal Interaction Interface for Mobile and Long-Lived Task Execution. In Proceedings of the ACM SIGCHI Symposium on Engineering Interactive Computing Systems (Valencia, Spain) (EICS '19). ACM, New York, NY, USA, Article 4, 5 pages. https://doi.org/10.1145/3319499.3328226

[61] Florian Heller, Davy Vanacken, Eva Geurts, and Kris Luyten. 2020. Impact of Situational Impairment on Interaction with Wearable Displays. In 22nd International Conference on Human-Computer Interaction with Mobile Devices and Services (Oldenburg, Germany) (MobileHCI '20). ACM, New York, NY, USA, Article 21, 5 pages. https://doi.org/10.1145/3406324.3410540

[62] Marc Herrlich, Anke V. Reinschluessel, Markus Willems, Nils Langhorst, David Black, Tanja Döring, Christian Rieder, Ron Kikinis, and Rainer Malaka. 2020. Put That Needle There: Customized Flexible On-Body Thin-Film Displays for Medical Navigation. ACM Trans. Comput. Heal. 1, 3 (2020), 16:1-16:17. https: //doi.org/10.1145/3386307

[63] Susanna Hertrich and Aiktoshi Honda. 2017. Brighter Than a Thousand Suns: A Uniform to Detect and Display Radiation. In Proceedings of the Eleventh International Conference on Tangible, Embedded, and Embodied Interaction (Yokohama, Japan) (TEI '17). ACM, New York, NY, USA, 659-663. https://doi.org/10.1145/3024969.3025055

[64] Thuong N. Hoang, Hasan Shahid Ferdous, Frank Vetere, and Martin Reinoso. 2018. Body as a Canvas: An Exploration on the Role of the Body as Display of Digital Information. In Proceedings of the 2018 on Designing Interactive Systems Conference 2018, DIS 2018, Hong Kong, China, June 09-13, 2018, Ilpo Koskinen, Youn-Kyung Lim, Teresa Cerratto Pargman, Kenny K. N. Chow, and William Odom (Eds.). ACM, New York, NY, USA, 253-263. https://doi.org/10.1145/ 3196709.3196724

[65] Ynvisible Interactive Inc. 2018. Electrochromic Displays. Website. https: //www.ynvisible.com

[66] Instructables 2018. Instructables - How to make anything. http://www. instructables.com

[67] Milena Iossifova and Younghui Kim. 2004. HearWear: The Fashion of Environmental Noise Display. In ACM SIGGRAPH 2004 Emerging Technologies (Los Angeles, California) (SIGGRAPH '04). ACM, New York, NY, USA, 7-. https://doi.org/10.1145/1186155.1186163

[68] Margot Jacobs and Linda Worbin. 2005. Reach: Dynamic Textile Patterns for Communication and Social Expression. In CHI '05 Extended Abstracts on Human Factors in Computing Systems (Portland, OR, USA) (CHI EA '05). ACM, New York, NY, USA, 1493-1496. https://doi.org/10.1145/1056808.1056949

[69] Pradthana Jarusriboonchai, Emmi Harjuniemi, Heiko Müller, Ashley Colley, and Jonna Häkkilä. 2019. Linn Dress: Enabling a Dynamically Adjustable Neckline. In Proceedings of the 23rd International Symposium on Wearable Computers 
(London, United Kingdom) (ISWC '19). ACM, New York, NY, USA, 274-278. https://doi.org/10.1145/3341163.3346934

[70] Pradthana Jarusriboonchai, Hong Li, Emmi Harjuniemi, Heiko Müller, and Jonna Häkkilä. 2020. Always with Me: Exploring Wearable Displays as a Lightweight Intimate Communication Channel. In TEI '20: Fourteenth International Conference on Tangible, Embedded, and Embodied Interaction, Sydney, NSW, Australia, February 9-12, 2020, Elise van den Hoven, Lian Loke, Orit Shaer, Jelle van Dijk, and Andrew L. Kun (Eds.). ACM, New York, NY, USA, 771-783. https://doi.org/10.1145/3374920.3375011

[71] Pradthana Jarusriboonchai, Emma Napari, Oskar Juhlin, and Jonna Häkkilä. 2019 Exploring Non-emissive Wearable Display As a Clothing Accessory. In Adjunct Proceedings of the 2019 ACM International Joint Conference on Pervasive and Ubiquitous Computing and Proceedings of the 2019 ACM International Symposium on Wearable Computers (London, United Kingdom) (UbiComp/ISWC'19 Adjunct). ACM, New York, NY, USA, 89-92. https://doi.org/10.1145/3341162.3343787

[72] Pradthana Jarusriboonchai, Thomas Olsson, Vikas Prabhu, and Kaisa VäänänenVainio-Mattila. 2015. CueSense: A Wearable Proximity-Aware Display Enhanc ing Encounters. In Proceedings of the 33rd Annual ACM Conference Extended Abstracts on Human Factors in Computing Systems (Seoul, Republic of Korea) (CHI EA '15). ACM, New York, NY, USA, 2127-2132. https://doi.org/10.1145/ 2702613.2732833

[73] Walther Jensen, Ashley Colley, and Markus Löchtefeld. 2019. VitaBoot: Footwear with Dynamic Graphical Patterning. In Proceedings of the 23rd International Symposium on Wearable Computers (London, United Kingdom) (ISWC '19). ACM, New York, NY, USA, 279-283. https://doi.org/10.1145/3341163.3346937

[74] Achim Kampker, Saskia Wessel, Nicolas Lutz, Matthias Bildhauer, and Martin Hehl. 2020. Holistic integration of a VR solution into the planning process of scalable production systems. Procedia CIRP 88 (2020), 133 - 138. https://doi.org/ 10.1016/j.procir.2020.05.024 13th CIRP Conference on Intelligent Computation in Manufacturing Engineering, 17-19 July 2019, Gulf of Naples, Italy.

[75] Cindy Hsin-Liu Kao, Bichlien Nguyen, Asta Roseway, and Michael Dickey. 2017 EarthTones: Chemical Sensing Powders to Detect and Display Environmenta Hazards Through Color Variation. In Proceedings of the 2017 CHI Conference Extended Abstracts on Human Factors in Computing Systems (Denver, Colorado, USA) (CHI EA '17). ACM, New York, NY, USA, 872-883. https://doi.org/10.1145 3027063.3052754

[76] Hsin-Liu Cindy Kao, Christian Holz, Asta Roseway, Andres Calvo, and Chris Schmandt. 2016. DuoSkin: Rapidly Prototyping On-skin User Interfaces Using Skin-friendly Materials. In Proceedings of the 2016 ACM International Symposium on Wearable Computers (ISWC '16). ACM, New York, NY, USA, 16-23. https: //doi.org/10.1145/2971763.2971777

[77] Hsin-Liu Cindy Kao, Manisha Mohan, Chris Schmandt, Joseph A Paradiso, and Katia Vega. 2016. ChromoSkin: Towards Interactive Cosmetics Using Thermochromic Pigments. In Proceedings of the 2016 CHI Conference Extended Abstracts on Human Factors in Computing Systems (CHI EA '16). ACM, New York, NY, USA, 3703-3706. https://doi.org/10.1145/2851581.2890270

[78] Thorsten Karrer, Moritz Wittenhagen, Leonhard Lichtschlag, Florian Heller, and Jan Borchers. 2011. Pinstripe: Eyes-free Continuous Input on Interactive Clothing. In Proceedings of the SIGCHI Conference on Human Factors in Computing Systems (Vancouver, BC, Canada) (CHI '11). ACM, New York, NY, USA 1313-1322. https://doi.org/10.1145/1978942.1979137

[79] Ruth Kikin-Gil. 2006. BuddyBeads: techno-jewelry for non-verbal communication within teenager girls groups. Pers. and Ubiqu. comp. 10, 2-3 (2006), 106-109. https://doi.org/10.1007/s00779-005-0015-x

[80] Konstantin Klamka, Tom Horak, and Raimund Dachselt. 2020. Watch+Strap: Extending Smartwatches with Interactive StrapDisplays. In Proceedings of the 2020 CHI Conference on Human Factors in Computing Systems (Honolulu, Hawaii, USA) (CHI '20). ACM, New York, NY, USA, 1-15. https://doi.org/10.1145/3313831. 3376199

[81] Rebecca Kleinberger and Alisha Panjwani. 2018. Digitally Enchanted Wear: A Novel Approach in the Field of Dresses As Dynamic Digital Displays. In Proceedings of the Twelfth International Conference on Tangible, Embedded, and Embodied Interaction (Stockholm, Sweden) (TEI '18). ACM, New York, NY, USA, 27-34. https://doi.org/10.1145/3173225.3173250

[82] Chelsea Klukas. 2019. Matrix Collection. https://www.lumencouture.com/ultrathin-led-matrix-fashiontech/.

[83] Ik Jang Ko, Jin Hwan Park, Gyeong Woo Kim, Raju Lampande, and Jang Hyuk Kwon. 2019. An optically efficient full-color reflective display with an electrochromic device and color production units. Fournal of Information Display 20, 3 (2019), 155-160. https://doi.org/10.1080/15980316.2019.1649310 arXiv:https://doi.org/10.1080/15980316.2019.1649310

[84] Ebru Kurbak and Irene Posch. 2015. 1-bit Textile. http://etextile-summercamp. org/swatch-exchange/1-bit-textile/.

[85] L!ber8. 2018. Tago Arc Bracelet. https://www.liber8tech.com.

[86] Hyein Lee, Yoonji Kim, and Andrea Bianchi. 2020. MAScreen: Augmenting Speech with Visual Cues of Lip Motions, Facial Expressions, and Text Using a Wearable Display. In SIGGRAPH Asia 2020 Emerging Technologies, SA 2020, Virtual Event, Republic of Korea, December 4-13, 2020. ACM, New York, NY, USA
2:1-2:2. https://doi.org/10.1145/3415255.3422886

[87] Mandy Leung, Martin Tomitsch, and Andrew Vande Moere. 2011. Designing a Personal Visualization Projection of Online Social Identity. In CHI '11 Extended Abstracts on Human Factors in Computing Systems (Vancouver, BC, Canada) (CHI EA '11). ACM, New York, NY, USA, 1843-1848. https://doi.org/10.1145/ 1979742.1979882

[88] Lilypad 2018. Lilypad - Sewable Electronics. https://www.sparkfun.com/ lilypad_sewable_electronics.

[89] Torsten Linz, Christine Kallmayer, Rolf Aschenbrenner, and Herbert Reichl. 2005. Embroidering electrical interconnects with conductive yarn for the integration of flexible electronic modules into fabric. In ISWC '05. Fraunhofer IZM, IEEE, New York, NY, USA, 86-89. https://doi.org/10.1109/ISWC.2005.19

[90] Xin LIU, Katia Vega, Jing Qian, Joseph Paradiso, and Pattie Maes. 2016. Fluxa: Body Movements As a Social Display. In Proceedings of the 29th Annual Symposium on User Interface Software and Technology (Tokyo, Japan) (UIST '16 Adjunct). ACM, New York, NY, USA, 155-157. https://doi.org/10.1145/2984751.2985741

[91] Joanne Lo, Doris Jung Lin Lee, Nathan Wong, David Bui, and Eric Paulos. 2016. Skintillates: Designing and Creating Epidermal Interactions. In Proceedings of the 2016 ACM Conference on Designing Interactive Systems (Brisbane, QLD, Australia) (DIS '16). ACM, New York, NY, USA, 853-864. https://doi.org/10. $1145 / 2901790.2901885$

[92] Bengt Lüers, Thomas Crone, Veronika Strokova, Jutta Fortmann, Susanne Boll, and Wilko Heuten. 2014. Illuminated Ring - A Wearable Display to Support Fluid Intake. In Mensch \& Computer 2014 - Tagungsband, 14. Fachübergreifende Konferenz für Interaktive und Kooperative Medien - Interaktiv unterwegs - Freiräume gestalten, 31. August - 3. September 2014, München, Germany, Andreas Butz, Michael Koch, and Johann H. Schlichter (Eds.). De Gruyter Oldenbourg, Oldenburg, Germany, 339-342. https://dl.gi.de/20.500.12116/7554

[93] Laura Lugaresi, Kaiyuan Lin, and Dingding Zheng. 2018. Wearable Aura: Interactive Personal Projection to Bring People Closer. In Extended Abstracts of the 2018 CHI Conference on Human Factors in Computing Systems (Montreal QC, Canada) (CHI EA '18). ACM, New York, NY, USA, SDC05:1-SDC05:6. https://doi.org/10.1145/3170427.3180651

[94] Lumos 2018. Lumos Helmet. https://lumoshelmet.co.

[95] Kris Luyten, Donald Degraen, Gustavo Rovelo Ruiz, Sven Coppers, and Davy Vanacken. 2016. Hidden in Plain Sight: An Exploration of a Visual Language for Near-Eye Out-of-Focus Displays in the Peripheral View. In Proceedings of the 2016 CHI Conference on Human Factors in Computing Systems (San Jose, California, USA) (CHI '16). ACM, New York, NY, USA, 487-497. https://doi.org/ $10.1145 / 2858036.2858339$

[96] Kent Lyons, Seung Wook Kim, Shigeyuki Seko, David Nguyen, Audrey Desjardins, Mélodie Vidal, David Dobbelstein, and Jeremy Rubin. 2014. Loupe: A Handheld Near-eye Display. In Proceedings of the 27th Annual ACM Symposium on User Interface Software and Technology (UIST '14). ACM, New York, NY, USA, 351-354. https://doi.org/10.1145/2642918.2647361

[97] Kent Lyons, David Nguyen, Daniel Ashbrook, and Sean White. 2012. Facet: A Multi-segment Wrist Worn System. In Proceedings of the 25th Annual ACM Symposium on User Interface Software and Technology (Cambridge, Massachusetts, USA) (UIST '12). ACM, New York, NY, USA, 123-130. https://doi.org/10.1145/ 2380116.2380134

[98] Hiroyuki Manabe and Yuki Iwai. 2020. Backhand Display: A Wearable Device for the Back of the Hand. In ISS '20: Interactive Surfaces and Spaces, Virtual Event, Portugal, November 8-11, 2020, Companion Volume, Joaquim A. Jorge, Alfredo Ferreira, Nicolai Marquardt, and Fanny Chevalier (Eds.). ACM, New York, NY, USA, 41-45. https://doi.org/10.1145/3380867.3426200

[99] Steve Mann. 2004. Continuous Lifelong Capture of Personal Experience with EyeTap. In Proceedings of the the 1st ACM Workshop on Continuous Archival and Retrieval of Personal Experiences (New York, New York, USA) (CARPE'04). ACM, New York, NY, USA, 1-21. https://doi.org/10.1145/1026653.1026654

[100] Steve Mann, James Fung, Chris Aimone, Anurag Sehgal, and Daniel Chen. 2005. Designing EyeTap digital eyeglasses for continuous lifelong capture and sharing of personal experiences. In alt.CHI '05 Extended Abstracts on Human Factors in Computing Systems (CHI EA '05). ACM, New York, NY, USA, 2202-2207.

[101] Steve Mann, Ryan E. Janzen, Hang Wu, Max Hao Lu, and Nitin Guleria. 2015. Bright ideas: A wearable interactive "Inventometer" (brainwave-based idea display). In 2015 IEEE Games Entertainment Media Conference, GEM 2015, Toronto, ON, Canada, October 14-16, 2015, Elena G. Bertozzi, Bill Kapralos, Nahum D. Gershon, and Jim R. Parker (Eds.). IEEE, New York, NY, USA, 1-8. https: //doi.org/10.1109/GEM.2015.7377239

[102] Matthew Mauriello, Michael Gubbels, and Jon E Froehlich. 2014. Social Fabric Fitness: The Design and Evaluation of Wearable E-textile Displays to Support Group Running. In Proceedings of the SIGCHI Conference on Human Factors in Computing Systems (CHI '14). ACM, New York, NY, USA, 2833-2842. https: //doi.org/10.1145/2556288.2557299

[103] Daniel C. McFarlane and Steven M. Wilder. 2009. Interactive Dirt: Increasing Mobile Work Performance with a Wearable Projector-Camera System. In Proceedings of the 11th International Conference on Ubiquitous Computing (Orlando, Florida, USA) (UbiComp '09). ACM, New York, NY, USA, 205-214. 
https://doi.org/10.1145/1620545.1620577

[104] Microsoft. 2015. HoloLens. https://www.microsoft.com/en-us/hololens

[105] Pranav Mistry and Pattie Maes. 2009. SixthSense: A Wearable Gestural Interface. In ACM SIGGRAPH ASIA 2009 Sketches (Yokohama, Japan) (SIGGRAPH ASIA '09). ACM, New York, NY, USA, Article 11, 1 pages. https://doi.org/10.1145/ 1667146.1667160

[106] Pranav Mistry, Pattie Maes, and Liyan Chang. 2009. WUW - Wear Ur World: A Wearable Gestural Interface. In CHI '09 Extended Abstracts on Human Factors in Computing Systems (Boston, MA, USA) (CHI EA '09). ACM, New York, NY, USA, 4111-4116. https://doi.org/10.1145/1520340.1520626

[107] Andrew Vande Moere and Monika Hoinkis. 2006. A Wearable Folding Display for Self-Expression. In Proceedings of the 18th Australia Conference on Computer-Human Interaction: Design: Activities, Artefacts and Environments (Sydney, Australia) (OZCHI '06). ACM, New York, NY, USA, 301-304. https: //doi.org/10.1145/1228175.1228228

[108] Heiko Müller, Ashley Colley, Jonna Häkkilä, Jonna, Walther Jensen, and Markus Löchtefeld. 2019. Using Electrochromic Displays to Display Ambient Information and Notifications. In Ubicomp Adjunct '19 (London, United Kingdom). ACM New York, NY, USA, 1075-1078. https://doi.org/10.1145/3341162.3344844

[109] Nike. 2012. Nike+ FuelBand. http://www.nike.com/us/en_us/c/nikeplusfuelband.

[110] Takuya Nojima, Miki Yamamura, Junichi Kanebako, Lisako Ishigami, Mage Xue, Hiroko Uchiyama, and Naoko Yamazaki. 2015. Bio-Collar: A Wearable Optic kinetic Display for Awareness of Bio-status. In Proceedings of the 6th Augmented Human International Conference (Singapore, Singapore) (AH '15). ACM, New York, NY, USA, 187-188. https://doi.org/10.1145/2735711.2735805

[111] North. 2018. Focals Smart Glasses. https://www.bynorth.com/focals

[112] Simon Olberding, Kian Peen Yeo, Suranga Nanayakkara, and Jurgen Steimle. 2013. AugmentedForearm: Exploring the Design Space of a Display-enhanced Forearm. In Proceedings of the 4th Augmented Human International Conference (Stuttgart, Germany) (AH '13). ACM, New York, NY, USA, 9-12. https://doi. org $/ 10.1145 / 2459236.2459239$

[113] Alex Olwal and Bernard Kress. 2018. 1D Eyewear: Peripheral, Hidden LEDs and Near-eye Holographic Displays for Unobtrusive Augmentation. In Proceedings of the 2018 ACM International Symposium on Wearable Computers (Singapore, Singapore) (ISWC '18). ACM, New York, NY, USA, 184-187. https://doi.org/10. $1145 / 3267242.3267288$

[114] Orphe. 2015. One - Footwear elevating performers to the next stage. https //orphe.shoes/.

[115] Maggie Orth. 2002. E-Ink Fashion Module. http://www.maggieorth.com/art FashionModule.html

[116] A. Oulasvirta, N. R. Dayama, M. Shiripour, M. John, and A. Karrenbauer. 2020 Combinatorial Optimization of Graphical User Interface Designs. Proc. IEEE 108, 3 (2020), 434-464.

[117] Mitchell Page and Andrew Vande Moere. 2007. Evaluating a Wearable Display Jersey for Augmenting Team Sports Awareness. In Pervasive Computing, Anthony LaMarca, Marc Langheinrich, and Khai N. Truong (Eds.). Springer, Berlin, Heidelberg, 91-108.

[118] Seonwook Park, Christoph Gebhardt, Roman Rädle, Anna Maria Feit, Hana Vrzakova, Niraj Ramesh Dayama, Hui-Shyong Yeo, Clemens N. Klokmose, Aaron Quigley, Antti Oulasvirta, and Otmar Hilliges. 2018. AdaM: Adapting Multi-User Interfaces for Collaborative Environments in Real-Time. In Proceedings of the 2018 CHI Conference on Human Factors in Computing Systems (Montreal QC, Canada) (CHI '18). ACM, New York, NY, USA, 1-14. https://doi.org/10.1145/ 3173574.3173758

[119] Patrick Parzer, Adwait Sharma, Anita Vogl, Jürgen Steimle, Alex Olwal, and Michael Haller. 2017. SmartSleeve: Real-time Sensing of Surface and Deformation Gestures on Flexible, Interactive Textiles, Using a Hybrid Gesture Detection Pipeline. In Proceedings of the 30th Annual ACM Symposium on User Interface Software and Technology (Québec City, QC, Canada) (UIST '17). ACM, New York, NY, USA, 565-577. https://doi.org/10.1145/3126594.3126652

[120] Roshan Lalintha Peiris, Adrian David Cheok, James Keng Soon Teh, Owen Noel Newton Fernando, Wen Yingqian, Andre Lim, Pan Yi, Doros Polydorou, Kian-Peng Ong, and Mili John Tharakan. 2009. AmbiKraf: an embedded nonemissive and fast changing wearable display. In ACM SIGGRAPH 2009 Emerging Technologies, SIGGRAPH '09, New Orleans, Louisiana, USA, August 3-7, 2009, Daniel Wigdor (Ed.). ACM, New York, NY, USA, 1:1. https://doi.org/10.1145/ 1597956.1597957

[121] Plastic Logic 2018. Plastic Logic 4.9" Lectum, Display Specifications. https://www.plasticlogic.com/products/displays/displays-with-epson/49-inch-display/.

[122] Henning Pohl, Justyna Medrek, and Michael Rohs. 2016. ScatterWatch: Subtle Notifications via Indirect Illumination Scattered in the Skin. In Proceedings of the 18th International Conference on Human-Computer Interaction with Mobile Devices and Services (Florence, Italy) (MobileHCI '16). ACM, New York, NY, USA 7-16. https://doi.org/10.1145/2935334.2935351

[123] Halley P Profita, James Clawson, Scott Gilliland, Clint Zeagler, Thad Starner Jim Budd, and Ellen Yi-Luen Do. 2013. Don't Mind Me Touching My Wrist:
A Case Study of Interacting with On-body Technology in Public. In ISWC '13. ACM, New York, NY, USA, 89-96. https://doi.org/10.1145/2493988.2494331

[124] Jing Qian, Meredith Young-Ng, Xiangyu Li, Angel Cheung, Fumeng Yang, and Jeff Huang. 2020. Portalware: A Smartphone-Wearable Dual-Display System for Expanding the Free-Hand Interaction Region in Augmented Reality. In Extended Abstracts of the 2020 CHI Conference on Human Factors in Computing Systems (CHI EA '20). ACM, New York, NY, USA, 1-8. https://doi.org/10.1145/3334480. 3383079

[125] Inka Rantala, Ashley Colley, and Jonna Häkkilä. 2018. Smart Jewelry: Augmenting Traditional Wearable Self-Expression Displays. In Proceedings of the 7th ACM International Symposium on Pervasive Displays (PerDis '18). ACM, New York, NY, USA, Article 22, 8 pages. https://doi.org/10.1145/3205873.3205891

[126] Majken K. Rasmussen, Esben W. Pedersen, Marianne G. Petersen, and Kasper Hornbæk. 2012. Shape-Changing Interfaces: A Review of the Design Space and Open Research Questions. In Proceedings of the SIGCHI Conference on Human Factors in Computing Systems (Austin, Texas, USA) (CHI '12). ACM, New York, NY, USA, 735-744. https://doi.org/10.1145/2207676.2207781

[127] Ringly 2018. Ringly. https://ringly.com.

[128] Paula Roinesalo, Tuomas Lappalainen, Ashley Colley, and Jonna Häkkilä. 2017. Breaking of the Dawn Jacket: Light in the Arctic Winter. In Proceedings of the 2017 ACM International Symposium on Wearable Computers (Maui, Hawaii) (ISWC '17). ACM, New York, NY, USA, 232-237. https://doi.org/10.1145/3123021.3123074

[129] Paula Roinesalo, Lasse Virtanen, Tuomas Lappalainen, Anu Kylmänen, and Jonna Häkkilä. 2016. Solar Shirt: Design of an Environmental Awareness Wearable. In Proceedings of the 2016 ACM International foint Conference on Pervasive and Ubiquitous Computing: Adjunct (Heidelberg, Germany) (UbiComp '16). ACM, New York, NY, USA, 495-499. https://doi.org/10.1145/2968219.2971350

[130] Jasna Rokegem. 2018. Jasna Rok - Fashion and Technology. http://www. jasnarok.com.

[131] Samsung. 2014. Fingers. https://news.samsung.com/global/samsungintroduces-its-first-wearable-glove-samsung-fingers.

[132] Samsung. 2018. Galaxy Watch. https://www.samsung.com/global/galaxy/ galaxy-watch/.

[133] Antti Sand and Ismo Rakkolainen. 2013. Mixed Reality with Multimodal Headmounted Pico Projector. In Proceedings of the Virtual Reality International Conference: Laval Virtual (Laval, France) (VRIC '13). ACM, New York, NY, USA, 14:1-14:2. https://doi.org/10.1145/2466816.2466831

[134] Meredith Scheff. 2010. The North Skirt. http://steampunkworkshop.com/northskirt/.

[135] Stefan Schneegass, Sophie Ogando, and Florian Alt. 2016. Using On-body Displays for Extending the Output of Wearable Devices. In Proceedings of the 5th ACM International Symposium on Pervasive Displays (Oulu, Finland) (PerDis '16). ACM, New York, NY, USA, 67-74. https://doi.org/10.1145/2914920.2915021

[136] Stefan Schneegass, Thomas Olsson, Sven Mayer, and Kristof Van Laerhoven. 2016. Mobile interactions augmented by wearable computing: A design space and vision. International fournal of Mobile Human Computer Interaction (IFMHCI) 8, 4 (2016), 104-114.

[137] Hasti Seifi, Farimah Fazlollahi, Michael Oppermann, John Andrew Sastrillo, Jessica Ip, Ashutosh Agrawal, Gunhyuk Park, Katherine J. Kuchenbecker, and Karon E. MacLean. 2019. Haptipedia: Accelerating Haptic Device Discovery to Support Interaction \& Engineering Design. In Proceedings of the 2019 CHI Conference on Human Factors in Computing Systems (Glasgow, Scotland Uk) (CHI '19). ACM, New York, NY, USA, 1-12. https://doi.org/10.1145/3290605.3300788

[138] Adhesh Shenoy, Mahathi Amencherla, Rahul Nagaraj, and T. S. Chandar. 2020. Optick - A Low Cost Wearable Head up Display for Search and Rescue Operations. In 11th International Conference on Computing, Communication and Networking Technologies (ICCCNT '20). IEEE, New York, NY, USA, 1-7. https://doi.org/10.1109/ICCCNT49239.2020.9225266

[139] ShiftWear 2018. ShiftWear Sneakers. https://www.shiftwear.com.

[140] Eun Kyung Shin. 2017. Hyperface. https://eunkyungshin.myportfolio.com/ enhanced-interface.

[141] Manlin Song and Katia Vega. 2018. HeartMe: Thermochromic Display As An Expression of Heart Health. In Proceedings of the 2018 ACM Conference Companion Publication on Designing Interactive Systems (Hong Kong, China) (DIS '18 Companion). ACM, New York, NY, USA, 311-314. https://doi.org/10. $1145 / 3197391.3205393$

[142] Timon Staal. 2016. TfCD Wearable Noise Display. Instructables. https://www. instructables.com/id/TfCD-Wearable-Noise-Display/.

[143] Paul Strohmeier, Jesse Burstyn, and Roel Vertegaal. 2015. Effects of Display Sizes on a Scrolling Task Using a Cylindrical Smartwatch. In Proceedings of the 17th International Conference on Human-Computer Interaction with Mobile Devices and Services Adjunct (Copenhagen, Denmark) (MobileHCI '15). ACM, New York, NY, USA, 846-853. https://doi.org/10.1145/2786567.2793710

[144] Chao-Huai Su, Liwei Chan, Chien-Ting Weng, Rong-Hao Liang, Kai-Yin Cheng, and Bing-Yu Chen. 2013. NailDisplay: Bringing an Always Available Visual Display to Fingertips. In Proceedings of the SIGCHI Conference on Human Factors in Computing Systems (CHI '13). ACM, New York, NY, USA, 1461-1464. https: //doi.org/10.1145/2470654.2466193 
[145] Yuta Sugiura, Toby Chong, Wataru Kawai, and Bruce H. Thomas. 2018. Public/Private Interactive Wearable Projection Display. In Proceedings of the 16th ACM SIGGRAPH International Conference on Virtual-Reality Continuum and Its Applications in Industry (Tokyo, Japan) (VRCAI '18). ACM, New York, NY, USA 10:1-10:6. https://doi.org/10.1145/3284398.3284415

[146] Ivan E. Sutherland. 1968. A Head-mounted Three Dimensional Display. In Proceedings of the December 9-11, 1968, Fall Joint Computer Conference, Part I (San Francisco, California) (AFIPS '68 (Fall, part I)). ACM, New York, NY, USA, 757-764. https://doi.org/10.1145/1476589.1476686

[147] Aneesh P. Tarun, Byron Lahey, Audrey Girouard, Winslow Burleson, and Roel Vertegaal. 2011. Snaplet: Using Body Shape to Inform Function in Mobile Flexible Display Devices. In CHI '11 Extended Abstracts on Human Factors in Computing Systems (Vancouver, BC, Canada) (CHI EA '11). ACM, New York, NY, USA, 329-334. https://doi.org/10.1145/1979742.1979701

[148] Kashyap Todi and Kris Luyten. 2014. Suit Up!: Enabling Eyes-free Interactions on Jacket Buttons. In CHI '14 Extended Abstracts on Human Factors in Computing Systems (Toronto, Ontario, Canada) (CHI EA '14). ACM, New York, NY, USA, 1549-1554. https://doi.org/10.1145/2559206.2581155

[149] Hung-Yu Tseng, Rong-Hao Liang, Liwei Chan, and Bing-Yu Chen. 2015. LEaD Utilizing Light Movement As Peripheral Visual Guidance for Scooter Navigation. In Proceedings of the 17th International Conference on Human-Computer Interaction with Mobile Devices and Services (Copenhagen, Denmark) (MobileHCI '15). ACM, New York, NY, USA, 323-326. https://doi.org/10.1145/2785830.2785831

[150] The Unseen. 2017. Fire - Thermochromic Hair Dye. http://seetheunseen.co.uk

[151] Katia Vega, Nan Jiang, Xin Liu, Viirj Kan, Nick Barry, Pattie Maes, Ali Yetisen, and Joe Paradiso. 2017. The Dermal Abyss: Interfacing with the Skin by Tattooing Biosensors. In Proceedings of the 2017 ACM International Symposium on Wearable Computers (Maui, Hawaii) (ISWC '17). ACM, New York, NY, USA, 138-145. https://doi.org/10.1145/3123021.3123039

[152] Inge Verboven, Jeroen Stryckers, Viktorija Mecnika, Glen Vandevenne, Manoj Jose, and Wim Deferme. 2018. Printing Smart Designs of Light Emitting Devices with Maintained Textile Properties. Materials 11, 2, Article 290 (2018), 10 pages. https://doi.org/10.3390/ma11020290

[153] Ulrich von Zadow, Wolfgang Büschel, Ricardo Langner, and Raimund Dachselt 2014. SleeD: Using a Sleeve Display to Interact with Touch-sensitive Display Walls. In Proceedings of the Ninth ACM International Conference on Interactive Tabletops and Surfaces (Dresden, Germany) (ITS '14). ACM, New York, NY, USA 129-138. https://doi.org/10.1145/2669485.2669507

[154] Zach Vorhies. 2016. Zackees Turn Signal Gloves. https://zackees.com/.

[155] Julie Wagner, Mathieu Nancel, Sean G Gustafson, Stéphane Huot, and Wendy E Mackay. 2013. Body-centric design space for multi-surface interaction. In Proceedings of the SIGCHI Conference on Human Factors in Computing Systems (CHI '13). ACM, New York, NY, USA, 1299-1308. https://doi.org/10.1145/2470654. 2466170

[156] Akira Wakita and Midori Shibutani. 2006. Mosaic Textile: Wearable Ambient Display with Non-emissive Color-changing Modules. In Proceedings of the 2006 ACM SIGCHI International Conference on Advances in Computer Entertainment Technology (Hollywood, California, USA) (ACE '06). ACM, New York, NY, USA 48-55. https://doi.org/10.1145/1178823.1178880

[157] Wouter Walmink, Alan Chatham, and Florian Mueller. 2014. Interaction Opportunities Around Helmet Design. In CHI '14 Extended Abstracts on Human Factors in Computing Systems (Toronto, Ontario, Canada) (CHI EA '14). ACM, New York, NY, USA, 367-370. https://doi.org/10.1145/2559206.2574803

[158] Wouter Walmink, Danielle Wilde, and Florian 'Floyd' Mueller. 2013. Displaying Heart Rate Data on a Bicycle Helmet to Support Social Exertion Experiences. In Proceedings of the 8th International Conference on Tangible, Embedded and Embodied Interaction (Munich, Germany) (TEI '14). ACM, New York, NY, USA 97-104. https://doi.org/10.1145/2540930.2540970

[159] Natalie Walsh. 2015. Fiber Optic Dress. Instructables. https://www.instructables. com/id/Fiber-Optic-Dress/.

[160] Wei Wang, Nick Bryan-Kinns, and Qifeng Yan. 2015. The design space and the shifting trigger in wearable product development. In International Design Congress (Gwangju, Korea).

[161] Yanan Wang, Shijian Luo, Yujia Lu, Hebo Gong, Yexing Zhou, Shuai Liu, and Preben Hansen. 2017. AnimSkin: Fabricating Epidermis with Interactive, Functional and Aesthetic Color Animation. In Proceedings of the 2017 Conference on Designing Interactive Systems (Edinburgh, United Kingdom) (DIS '17). ACM, New York, NY, USA, 397-401. https://doi.org/10.1145/3064663.3064687

[162] Martin Weigel, Aditya Shekhar Nittala, Alex Olwal, and Jürgen Steimle. 2017. SkinMarks: Enabling Interactions on Body Landmarks Using Conformal Skin Electronics. In Proceedings of the 2017 CHI Conference on Human Factors in Computing Systems (Denver, Colorado, USA) (CHI '17). ACM, New York, NY USA, 3095-3105. https://doi.org/10.1145/3025453.3025704

[163] Dirk Wenig, Johannes Schöning, Alex Olwal, Mathias Oben, and Rainer Malaka 2017. WatchThru: Expanding Smartwatch Displays with Mid-air Visuals and Wrist-worn Augmented Reality. In Proceedings of the 2017 CHI Conference on Human Factors in Computing Systems (Denver, Colorado, USA) (CHI '17). ACM, New York, NY, USA, 716-721. https://doi.org/10.1145/3025453.3025852
[164] Michael Wessely, Theophanis Tsandilas, and Wendy E. Mackay. 2016. Stretchis: Fabricating Highly Stretchable User Interfaces. In Proceedings of the 29th Annual Symposium on User Interface Software and Technology (Tokyo, Japan) (UIST '16). ACM, New York, NY, USA, 697-704. https://doi.org/10.1145/2984511.2984521

[165] Amanda Williams, Shelly Farnham, and Scott Counts. 2006. Exploring Wearable Ambient Displays for Social Awareness. In CHI'06 Extended Abstracts on Human Factors in Computing Systems (Montr\&\#233;al, Qu\&\#233;bec, Canada) (CHI EA '06). ACM, New York, NY, USA, 1529-1534. https://doi.org/10.1145/1125451. 1125731

[166] Graham Wilson, Martin Halvey, Stephen A. Brewster, and Stephen A. Hughes. 2011. Some Like It Hot: Thermal Feedback for Mobile Devices. In Proceedings of the SIGCHI Conference on Human Factors in Computing Systems (Vancouver, BC, Canada) (CHI '11). ACM, New York, NY, USA, 2555-2564. https://doi.org/10. 1145/1978942.1979316

[167] Raphael Wimmer and Florian Echtler. 2013. Exploring the Benefits of Fingernail Displays. In CHI '13 Extended Abstracts on Human Factors in Computing Systems (CHI EA '13). ACM, New York, NY, USA, 937-942. https://doi.org/10.1145/ 2468356.2468524

[168] Withings. 2017. Steel HR. https://www.withings.com/uk/en/steel-hr.

[169] Yunyun Wu, Sara S. Mechael, Yiting Chen, and Tricia Breen Carmichael. 2018. Solution Deposition of Conformal Gold Coatings on Knitted Fabric for E-Textiles and Electroluminescent Clothing. Advanced Materials Technologies 3, 3 (2018), 1700292. https://doi.org/10.1002/admt.201700292 arXiv:https://onlinelibrary.wiley.com/doi/pdf/10.1002/admt.201700292

[170] Robert Xiao, Teng Cao, Ning Guo, Jun Zhuo, Yang Zhang, and Chris Harrison. 2018. LumiWatch: On-Arm Projected Graphics and Touch Input. In Proceedings of the 2018 CHI Conference on Human Factors in Computing Systems (Montreal QC, Canada) (CHI '18). ACM, New York, NY, USA, 1-11. https://doi.org/10. $1145 / 3173574.3173669$

[171] Cheng Xu and Kent Lyons. 2015. Shimmering Smartwatches: Exploring the Smartwatch Design Space. In Proceedings of the Ninth International Conference on Tangible, Embedded, and Embodied Interaction (Stanford, California, USA) (TEI '15). ACM, New York, NY, USA, 69-76. https://doi.org/10.1145/2677199. 2680599

[172] Ungyeon Yang and Ki-Hong Kim. 2015. Optical see-through wearable display for close range interaction. In IEEE International Conference on Consumer Electronics (ICCE '15). IEEE, New York, NY, USA, 116-117. https://doi.org/10.1109/ICCE. 2015.7066343

[173] Zebra. 2018. WT6000 Wearable Computer. https://www.zebra.com/us/en/ products/mobile-computers/wearable-computers/wt6000.html. 